Basic Concepts of Kinematic-Wave Models

U. S. GEOLOGICAL SURVEY PROFESSIONAL PAPER 1302

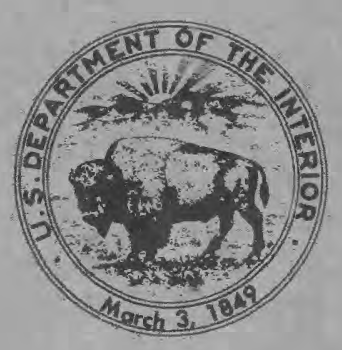




\section{Basic Concepts of Kinematic-Wave Models}

BY JEFFREY E. MILLER

U. S. GEOLOGICAL SURVEY PROFESIONAL PAPER 1302

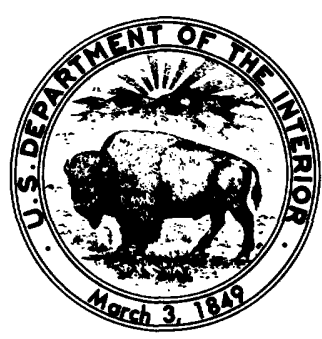




\section{UNITED STATES DEPARTMENT OF THE INTERIOR \\ WILLIAM P. CLARK, Secretary}

GEOLOGICAL SURVEY

Dallas L. Peck, Director

Library of Congress Cataloging in Publication Data

Miller, Jeffrey E.

Basic concepts of kinetic-wave models.

(Geological Survey professional paper ; 1302)

Bibliography: $p$.

Supt. of Docs. no.: I 19.16:1302

1. Water waves-Mathematical models. I. Title.

II. Series.

$\begin{array}{llll}\text { TC172.M54 } & 1983 & 627 ' .042 & 83-600251\end{array}$

For sale by the Distribution Branch, U.S. Geological Survey, 604 South Pickett Street, Alexandria, VA 22304 


\section{PREFACE}

Kinematic-wave models are used extensively by the U.S. Geological Survey. Both kinematic- and modified kinematic-wave models are used for channel and overland-flow routing in the Precipitation-Runoff Modeling system and in the Distributed Routing Rainfall-Runoff Model. The development of the theory and application of kinematic waves is complex, but it is not readily available in any one text. Therefore, the purpose of this report is to present the basic concepts of the kinematic-wave approximation of one-dimensional dynamic waves. The report is intended for an audience of field hydrologists applying kinematicwave models in practical situations such as watershed models. This report does not propose to assess or describe the current state of the art of approximate hydraulic-flow routing techniques. It is intended to be used as a basic reference on the theory and application of kinematic-wave models and modified kinematic-wave models.

The kinematic-wave model is one of a number of approximations of the dynamic-wave model. Because of the numerous approximations made in the development of the models and the difficulty involved in applying the solution techniques, the kinematic-wave approximation needs to be understood in relation to the other models. Therefore, a significant part of the report describes the dynamic-wave model, including a detailed development of the governing equations. This development is important because it shows the assumptions made in describing unsteady flow with the full dynamic-wave equations. These assumptions are inherent in any of the models that are approximations of the dynamic-wave model, such as the kinematic-wave model.

Because of the intended audience, the equation developments are given in detail so that they will be understood as easily as possible. There are many basic concepts involved in the application of kinematic-wave models that can be confusing. Instead of assuming that these concepts are obvious to the reader, they are included in a supplemental information section. 


\section{.}




\section{CONTENTS}

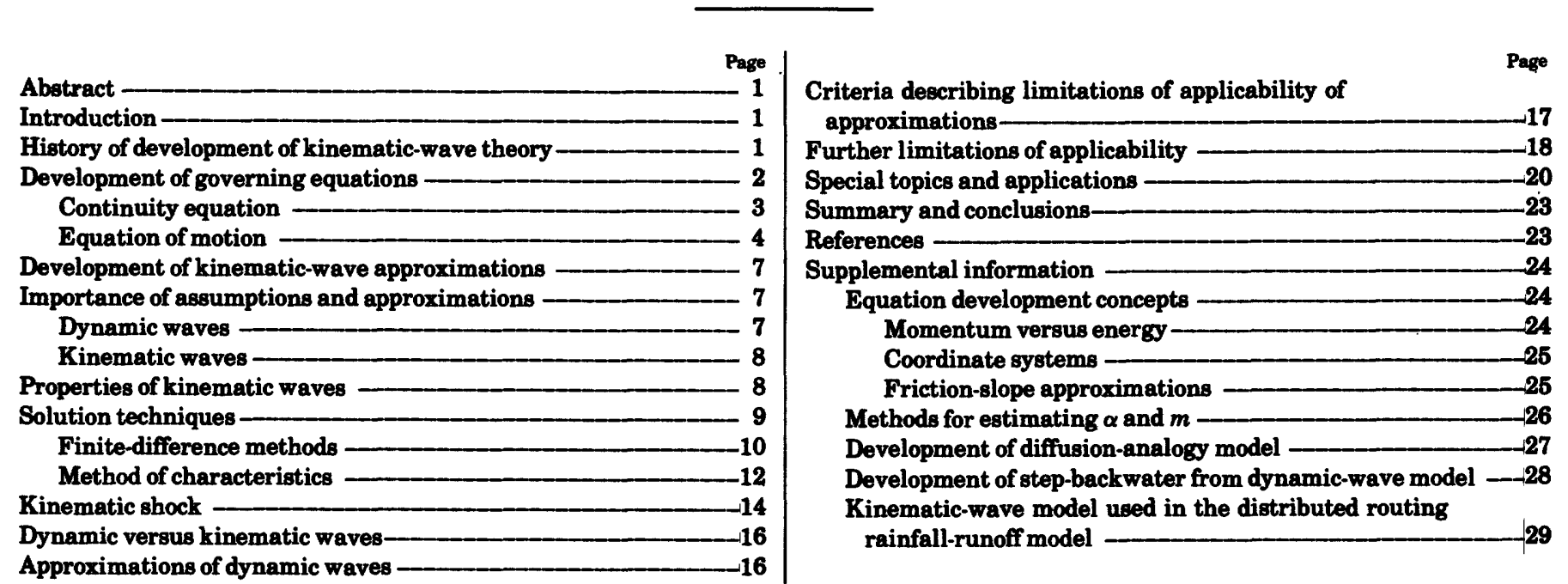

\section{ILLUSTRATIONS}

Froure 1. Definition sketch for the continuity equation

Page

Longitudinal section showing prism and wedge storage ___

3. Definition sketches for the equation of motion - 5

4. Water-surface profiles at time, $t$ and $t+\Delta t$, showing distortion or steepening of the kinematic wave _-_

5. Fixed grid in the $x-t$ plane showing location of grid points and counters, $i$ and $j$

6. Schematic of $x-t$ plane showing typical characteristic paths for the kinematic-wave equations, with and without lateral inflow-

7. Water-surface profiles at time, $t$ and $t+\Delta t$, and the characteristic paths between them showing the condition necessary for shock formation

8. Comparison of kinematic- and dynamic-wave model results showing the upstream hydrograph and predicted hydrographs at $20,000,40,000,60,000$, and 80,000 feet

9. Four-point finite-difference grid

10. Use of $\log -\log$ plot of discharge, $Q$, and flow area, $A$, to define the routing parameters, $\alpha$ and $m$ 


\section{TABLE}

TABLE 1. RELATIONS FOR ESTIMATING $\alpha$ and $m$ based on physical characteristics of channel segments

Page

TABLE 1. RELATIONS FOR ESTIMATING $\alpha$ and $m$ based on physical characteristics of chann

\section{SELECTED FACTORS FOR CONVERTING INCH-POUND UNITS TO THE INTERNATIONAL SYSTEM OF UNITS (SI)}

For those readers who may prefer to use the International System of units (SI) rather than inchpound units, the conversion factors for the terms used in this report are given below.

Multiply inch-pound unit

Cubic foot per second

Foot

Foot per mile

Foot per second
By

0.02832

0.3048

0.1894

0.3048
To obtain SI unit

cubic meter per second

meter

meter per kilometer

meter per second

\section{DEFINITION OF SYMBOLS}

$\boldsymbol{a}$ acceleration or coefficient in the wetted perimeter-flow area relationship,

A cross-sectional flow area,

$A_{f}$ area of flow at pipe-full conditions,

b coefficient in the wetted perimeter flow-area relationship, water-surface width,

wave celerity,

C Chézy friction coefficient or Courant number,

$f$ force,

$F$ Froude number $=v / \sqrt{g y}$,

$F_{o} \quad$ Froude number for normal flow $=v_{o} / \sqrt{g} y_{o}$,

g gravitational acceleration,

$h$ height of water surface above datum or piezometric head,

$h_{f}$ friction loss,

$H$ total energy head,

$i$ finite-difference grid location in the $x$-direction,

$j$ finite-difference grid location in the time direction,

$\boldsymbol{K}$ kinematic-flow number,

$L$ length of wave,

$L_{o} \quad$ length of channel segment or overland-flow plane,

$m$ mass or coefficient in the steady uniform flow equation approximation,

$n$ Manning's roughness coefficient,

$P$ wetted perimeter,

$P_{c}$ channel parameter, $q$ discharge per unit width (can be either flow in a channel or lateral inflow),

$\boldsymbol{Q}$ discharge,

$\boldsymbol{Q}_{f}$ discharge at pipe-full conditions,

$R$ hydraulic radius $=A / P$,

$S_{a}$ acceleration slope,

$S_{e}$ energy slope $=-\mathrm{d} H / \mathrm{d} x$,

$S_{f}$ friction slope,

$S_{o}$ bed slope $=-\mathrm{d} z / \mathrm{d} x$,

$t$ time,

$T$ wave period,

$v$ flow velocity,

$v_{o}$ steady-state normal flow velocity,

$W_{t}$ time derivative weighting factor,

$W_{x}$ spacial derivative weighting factor,

$x$ horizontal distance,

$y$ vertical stream depth,

$y_{0}$ normal depth,

$z$ height of stream bottom above datum,

$\alpha$ coefficient in the steady uniform flow equation approximation,

$\gamma$ specific weight of water,

$\theta$ angle of the channel bed with the horizontal,

$\mu$ diffusion coefficient,

Q density of fluid, and

$\tau_{o}$ mean longitudinal shear stress. 


\title{
BASIC CONCEPTS OF KINEMATIC-WAVE MODELS
}

\author{
By JefFrey E. Miller
}

\begin{abstract}
The kinematic-wave model is one of a number of approximations of the dynamic-wave model. The dynamic-wave model describes onedimensional shallow-water waves (unsteady, gradually varied, openchannel flow). This report provides a basic reference on the theory and applications of the kinematic-wave model and describes the limitations of the model in relation to the other approximations of the dynamic-wave model. In the kinematic-wave approximation, a number of the terms in the equation of motion are assumed to be insignificant. The equation of motion is replaced by an equation describing uniform flow. Thus, the kinematic-wave model is described by the continuity equation and a uniform-flow equation such as the wellknown Chézy or Manning formulas. Kinematic-wave models are applicable to overland flow where lateral inflow is continuously added and is a large part of the total flow. For channel-routing applications, the kinematic-wave model always predicts a steeper wave with less dispersion and attenuation than actually occurs. The effect of the accumulation of errors in the kinematic-wave model shows that the approximations made in the development of the kinematic-wave equations are not generally justified for most channel-routing applications. Modified flow-routing models can be used which help to stop the accumulation of errors that occur when the kinematic-wave model is applied.
\end{abstract}

\section{INTRODUCTION}

The kinematic-wave model is one of a number of approximations of the dynamic-wave model. The dynamicwave model describes one-dimensional shallow-water waves (unsteady, gradually varied, open-channel flow) and consists of the continuity equation and the equation of motion with appropriately prescribed initial and boundary conditions. In most approximations of dynamic waves, the continuity equation and an approximation of the equation of motion are solved. In the kinematic-wave approximation, a number of the terms in the equation of motion are assumed to be insignificant; therefore, the equation of motion simply states that the friction slope is equal to the bed slope. It is replaced by an equation describing uniform (which also implies steady) flow. Thus, the kinematic-wave model is described by the continuity equation and a uniformflow equation, such as the well-known Chézy or Manning formulas, plus the usually imposed initial and boundary conditions.
Kinematic-wave theory describes a distinctive type of wave motion that can occur in many one-dimensional flow problems (Lighthill and Whitham, 1955, p. 281). The theory is described in this report as an approximation of dynamic-wave theory applied to water-routing problems.

The purpose of this report is to provide a basic reference on the theory and applications of the kinematicwave model and to describe the limitations of the model in relation to the dynamic-wave model. The kinematic-wave model is also described in relation to the other hydraulic-routing techniques that are approximations of the dynamic-wave model. Unless specifically indicated, the channels described in this report are prismatic, and most of the explanations of the behavior of the models will use examples that include no lateral inflow. These simplifications make the behavior of the models easier to illustrate because changes in numerically routed hydrographs are not masked by other influences. The development, assumptions and approximations, properties, criteria for application, current applications, and solution techniques of kinematic-wave theory in water routing will be examined.

\section{HISTORY OF DEVELOPMENT OF KINEMATIC-WAVE THEORY}

The development of kinematic-wave theory occurred late in the history of the development of the theory of open-channel flow. It is based on the early developments in the study of steady varied flow and the later developments in the study of unsteady flow.

According to Bakhmeteff (1932, p. 299), the theory of steady varied flow is usually associated with $\mathrm{J}$. M. Belanger. Bakhmeteff stated that Belanger, in his 1828 paper, covers the "subject of varied flow in a remarkably complete and comprehensive manner." Bakhmeteff listed earlier references on varied flow, such as Dubuat in 1779, Venturoli in 1818, and particularly Massetti in 1827. He also stated that Poncelet is believed to have developed the equation of varied 
flow about the same time as Belanger and that Euler developed the foundations of hydromechanics in 1755. Bakhmeteff further stated that "while Belanger and his followers deducted the varied flow equation from the general Newtonian equation of motion," Coriolis in 1836 "made use of the principle of conservation of energy and thus was the first to suggest the reasoning which since has been followed in textbooks on hydraulics in establishing the so-called Bernoulli equation."

According to Yevjevich (1975, p. 2-5), the study of unsteady flow started with two French mathematicians-Laplace, in 1775, and Lagrange, in 1781. The Lagrange celerity formula was reported in 1788, providing impetus for further studies. Barre de Saint Venant presented the equation of motion and the water-continuity equation in 1871 . These equations are now known as the Saint Venant partial-differential equations of unsteady flow. Also in 1871, Saint Venant attempted to integrate the continuity equation and the equation of motion by setting the channel slope equal to the energy line. This approach is similar to kinematic-wave theory.

Lighthill and Whitham (1955) stated that several writers independently have given the theory of continuous kinematic waves as applied to flood movement. Complete treatments were given by Boussinesq in 1877 and by Forcheimer in 1930 (in his book $H y d r a u l i k)$. These two authors referred to an unpublished report by Kleitz in 1858, Breton in 1867, and Graeff in 1875 as pioneers of the theory. Lighthill and Whitham also stated that Seddon, in 1900 discussed the problem with reference to the Mississippi and its tributaries and that Seddon, although unaware of the earlier work, showed an understanding of the variety of mechanism that govern the relationship between flow area and discharge.

The principal theoretical work on kinematic waves was done by Lighthill and Whitham (1955). They named the theory "kinematic wave" and investigated the general properties of waves and shock waves based on the theory. They also provided a detailed treatment of the relationship between kinematic- and dynamicwave movement in rivers.

Application of kinematic-wave theory to channel routing has been described by Weinmann and Laurenson (1979), Henderson (1963), and Brakensiek (1967). Cunge (1969) showed that the Muskingum routing method can be considered a finite-difference approximation of the kinematic-wave model. Thus, kinematicwave theory has been widely applied to river routing for a number of years. The theory also has been applied to the problems of water routing in irrigation systems (see, for example, Cunge and Woolhiser, 1975) and storm water runoff (selected references are the Storm
Water Management Model, U.S. Environmental Protection Agency, 1971, and Dawdy and others, 1978).

Overland flow is viewed as wide shallow-channel flow and is often analyzed on a unit width basis with lateral inflow originating from rainfall excess. Henderson (1966, p. 394) stated that Japanese engineers applied kinematic-wave theory to the problem of overland runoff. Since then, a large amount of work has been done on the overland-flow problem. Selected examples are Henderson and Wooding (1964), Woolhiser and Liggett (1967), Kibler and Woolhiser (1970), Schaake (1970), Li and others (1975), and Borah and others (1980).

Many numerical solution techniques have been developed to solve the kinematic-wave equations. Usually these techniques involve finite-difference (see, for example, Kibler and Woolhiser, 1970) or method of characteristics (see, for example, Borah and others, 1980) Schemes. Cunge (1969), the U.S. Environmental Protection Agency (1971), and $\mathrm{Li}$ and others (1976) have applied modified kinematic-wave approximations in which adjusted slope terms are used or controlled numerical dispersion is added to the solution for channel-routing problems. In the time since the paper by Lighthill and Whitham was written, a large amount of work has been done in solving the kinematic-wave equations, applying the theory to channel and overland flow, determining when the theory is applicable, and describing the properties of waves based on the theory and solution techniques.

\section{DEVELOPMENT OF GOVERNING EQUATIONS}

The equations describing unsteady open-channel flow are a continuity equation and an equation of motion. The continuity equation is formulated based on the principle of conservation of mass. The principle states that the difference between the rates of inflow and outflow is equal to the rate of change in storage.

The development of the equation of motion involves Newton's second law and the principles of conservation of energy and momentum. Newton's second law is

$$
f=m a,
$$

where

$$
\begin{aligned}
& f=\text { force, } \\
& m=\text { mass, }
\end{aligned}
$$$$
\text { and }
$$$$
a=\text { acceleration. }
$$

The equation of motion can be derived from equation 1 directly, or it can be derived using conservation of energy or momentum concepts. The development of the 
continuity equation and the equation of motion used in this report follows Henderson (1966). The treatment given by Henderson is developed in detail and the equation of motion is based directly on Newton's second law instead of on the energy or momentum forms. It uses an orthogonal gravity-oriented coordinate system. The relationship between momentum and energy and a description of commonly used coordinate systems are described in the section "Supplemental Information" under "Equation Development Concepts."

The following references give equation developments for both the continuity equation and the equation of motion. Derivation of the equation of motion, based on the principle of conservation of energy, is given by Gilcrest (1950) and Chow (1959). The derivation of the equation of motion, based on the principle of conservation of momentum, is given by Liggett (1975), Theurer (1975), and Viessman and others (1977).

\section{CONTINUITY EQUATION}

The development of the continuity equation involves the following variables:

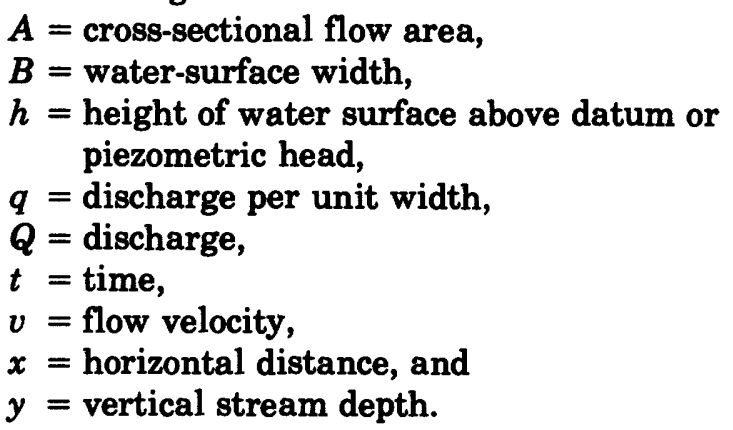

Consider a control volume enclosed by the water surface, the channel boundary surface, and two cross sections separated by a small distance, $\Delta x$, as shown in figure 1. Without lateral inflow or outflow.

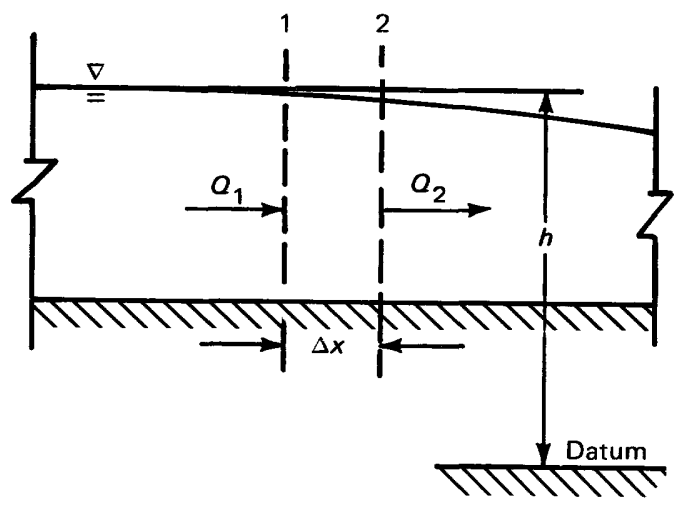

FIGURE 1.-Definition sketch for the continuity equation.

$$
Q_{2}-Q_{1}=\frac{\partial Q}{\partial x} \Delta x
$$

The right-hand side will be positive if the volume is decreasing. ( $Q$ may be changing with time as well as with $x$.)

Considering the change in water-surface level, the volume of water is changing at the rate of

$$
\frac{\partial h}{\partial t}(B \Delta x) \text {. }
$$

This term will be positive if the volume is increasing. Equations 2 and 3 must be equal in magnitude and opposite in sign. Therefore, an expression of continuity is

$$
\frac{\partial Q}{\partial x} \Delta x+\frac{\partial h}{\partial t}(B \Delta x)=0 .
$$

Dividing by $\Delta x$ yields

$$
\frac{\partial Q}{\partial x}+B \frac{\partial h}{\partial t}=0
$$

Equation 5 is the unsteady continuity equation. A form of equation 5 for more general cross-section shapes (where $\frac{\partial A}{\partial t}$ is substituted for $B \frac{\partial h}{\partial t}$ ) is

$$
\frac{\partial Q}{\partial x}+\frac{\partial A}{\partial t}=0 \text {. }
$$

Two additional alternative forms are developed as follows. When a channel is prismatic and rectangular, $Q=B q$. Substituting into equation 5 and dividing by $B$, the first alternative form is

$$
\frac{\partial q}{\partial x}+\frac{\partial h}{\partial t}=0
$$

The second alternative form, which is useful for visualizing each term, can be written by substituting $\frac{\partial Q}{\partial x}=\frac{\partial(A v)}{\partial x}$ and depth, $y$, for $h$ in equation 5 :

$$
\frac{\partial(A v)}{\partial x}+B \frac{\partial y}{\partial t}=0
$$

or

$$
A \frac{\partial v}{\partial x}+v \frac{\partial A}{\partial x}+B \frac{\partial y}{\partial t}=0
$$


The three terms in the form of the continuity equation given above are known as prism storage, wedge storage, and rate of rise terms. The physical meaning of the first two terms is shown in figure 2. Equation 9 is valid only for prismatic channels.

\section{EQUATION OF MOTION}

The development of the equation of motion involves the variables used in the continuity equation and the following additional variables:

$a=$ acceleration,

$C=$ Chezy friction coefficient,

$f=$ force,

$H=$ total energy head,

$\boldsymbol{g}=$ gravitational acceleration,

$m=$ mass,

$P=$ wetted perimeter,

$R=$ hydraulic radius $=A / P$,

$S_{a}=$ acceleration slope,

$S_{e}=$ energy slope $=-\mathrm{d} H / \mathrm{d} x$,

$S_{f}=$ friction slope,

$S_{o}=$ bed slope $=-\mathrm{d} z / \mathrm{d} x$,

$z=$ height of stream bottom above datum,

$\gamma=$ specific weight of water,

$\theta=$ angle of the channel bed with the horizontal,

$\rho=$ density of fluid, and

$\tau_{o}=$ mean longitudinal shear stress.

To develop the equation of motion, the forces on a fluid element in a channel section will be determined based in assumptions appropriate to one-dimensional shallowwater waves. Then the remaining acceleration and mass terms will be developed and substituted into Newton's second law.

Considering the channel sections shown in figure 3 and assuming that the pressure distribution is hydrostatic, the total horizontal hydrostatic pressure on the element shown is equal to the difference in the two hydrostatic pressures, $-\gamma \Delta h$, over the area of the element. The opposite sign is used because a negative $\Delta h$

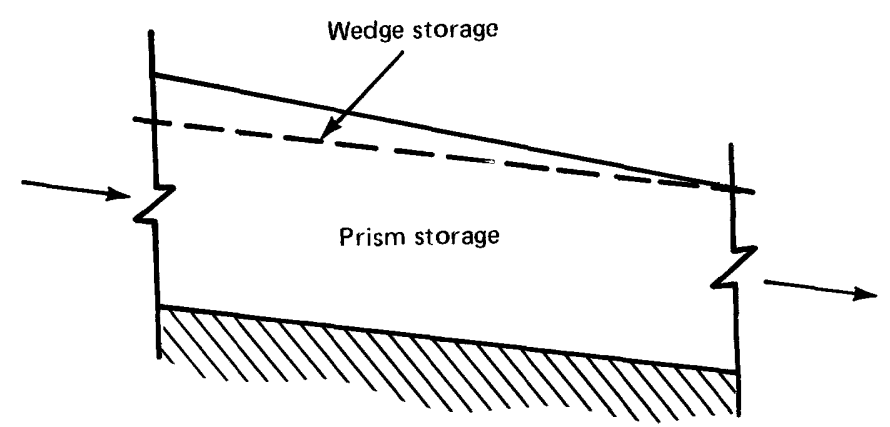

Figure 2.-Longitudinal section showing prism and wedge storage. will produce flow in the positive $x$-direction. The crosssectional flow area of the element is

$$
A=\Delta B y .
$$

There is only a small variation in area across $\Delta x$ if $\Delta h / y$ and $\Delta z / y$ are small. The hydrostatic force difference is then

$$
-\gamma A \Delta h .
$$

This force is resisted by a shear force equal to $\tau_{o}$ over the wetted perimeter along $\Delta x$ at an angle $\theta$ to the horizontal. The net force in the direction of the flow is

$$
f=-\gamma A \Delta h-\tau_{o} P \Delta x \cos \theta .
$$

Following the assumption of small slopes, $\cos \theta \cong 1$; therefore, equation 12 can be written

$$
f=-\gamma A \Delta h-\tau_{o} P \Delta x .
$$

This is the force term in Newton's second law.

Again, considering the channel section in figure 3, the mass term in Newton's second law is

$$
m=\varrho A \Delta x
$$

The acceleration term in Newton's second law is developed as follows. Noting that $a=\mathrm{d} v / \mathrm{d} t$, from the theory of partial differentiation,

$$
\frac{\mathrm{d} v}{\mathrm{~d} t}=\frac{\mathrm{d} x}{\mathrm{~d} t} \frac{\partial v}{\partial x}+\frac{\partial v}{\partial t} .
$$

Equation 15 describes the rate at which the velocity $v$ will change in the eyes of an observer moving in the direction $x$ with velocity $\mathrm{d} x / \mathrm{d} t$. If $\mathrm{d} v / \mathrm{d} t$ is to be interpreted as a fluid acceleration, the observer must be moving with the fluid itself. Thus, $\mathrm{d} x / \mathrm{d} t=v$ and

$$
a=\frac{\mathrm{d} v}{\mathrm{~d} t}=v \frac{\partial v}{\partial x}+\frac{\partial v}{\partial t} .
$$

Substituting equations 13,14 , and 16 into Newton's second law (eq 1) results in the following:

$$
-\gamma A \Delta h-\tau_{o} \mathrm{P} \Delta x=\varrho A \Delta x\left(v \frac{\partial v}{\partial x}+\frac{\partial v}{\partial t}\right) .
$$

Rearranging,

$$
\tau_{0}=\frac{-\varrho A \Delta x\left(v \frac{\partial v}{\partial x}+\frac{\partial v}{\partial t}\right)-\gamma A \Delta h}{P \Delta x} .
$$




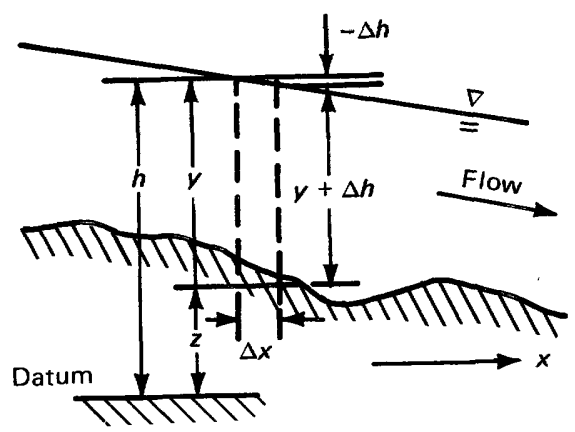

LONGITUDINAL SECTION

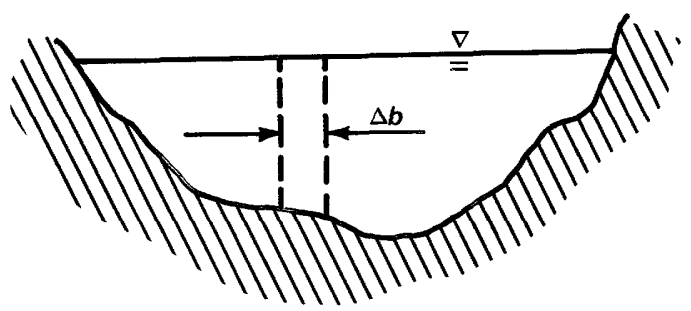

CROSS SECTION

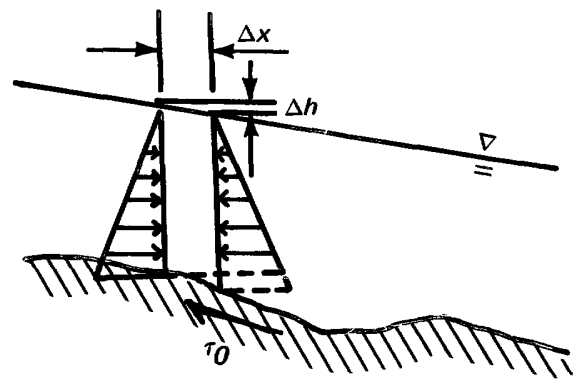

HYDROSTATIC PRESSURE DIAGRAN'US

Figure 3.-Definition sketches for the equation of motion.

Setting $A / P=R$ and taking the limit as $\Delta x \rightarrow 0$ so that $\frac{\Delta h}{\Delta x}=\frac{\partial h}{\partial x}$,

$$
\tau_{o}=-\varrho R\left(v \frac{\partial v}{\partial x}+\frac{\partial v}{\partial t}\right)-\gamma R \frac{\partial h}{\partial x}
$$

or, noting that $\gamma=\mathrm{eg}$,

$$
\tau_{o}=-\gamma R\left(\frac{v}{g} \frac{\partial v}{\partial x}+\frac{1}{g} \frac{\partial v}{\partial t}+\frac{\partial h}{\partial x}\right) .
$$

At this point, before the equation of motion can be developed further, three basic relationships-two for the friction slope and one for the longitudinal shear stress-must be introduced. The definition of the total energy head is

$$
H=h+\frac{v^{2}}{2 g} .
$$

This definition ignores the velocity distribution coefficient. This is often done in unsteady flow models because the coefficient is only of secondary importance (see Henderson, 1966, p. 21). The velocity distribution coefficients for both energy and momentum are ignored in this report unless specifically stated otherwise. Taking the partial derivative with respect to the downstream direction, the definition of the friction slope is

$$
S_{f}=\frac{-\partial H}{\partial x}=\frac{-\partial\left(h+\frac{v^{2}}{2 g}\right)}{\partial x}
$$

or, by the rules of differentiation,

$$
S_{f}=\frac{-\partial H}{\partial x}=-\left(\frac{\partial h}{\partial x}+\frac{v}{2 g} \frac{\partial v}{\partial x}+\frac{v}{2 g} \frac{\partial v}{\partial x}\right)
$$

or

$$
S_{f}=\frac{-\partial H}{\partial x}=-\left(\frac{\partial h}{\partial x}+\frac{v}{g} \frac{\partial v}{\partial x}\right) .
$$

The two remaining relationships are approximations when applied to the unsteady nonuniform flow situation. The derivation of these relationships is shown in the section "Supplemental Information" under "Equa- 
tion Development Concepts." They are the friction slope based on the Chézy equation,

$$
S_{f}=\frac{v^{2}}{C^{2} R}
$$

and the longitudinal shear stress expressed using the Chézy equation,

$$
\tau_{o}=\gamma R\left(\frac{v^{2}}{C^{2} R}\right) \text {. }
$$

Continuing the development of the equation of motion, equation 24 is substituted into equation 20:

$$
\tau_{o}=-\gamma R\left(\frac{\partial H}{\partial x}+\frac{1}{g} \frac{\partial v}{\partial t}\right)
$$

Setting equation 26 equal to equation 27 ,

$$
\gamma R \frac{v^{2}}{C^{2} R}=-\gamma R\left(\frac{\partial H}{\partial x}+\frac{1}{g} \frac{\partial v}{\partial t}\right)
$$

Rearranging,

$$
\frac{\partial H}{\partial x}+\frac{1}{g} \frac{\partial v}{\partial t}+\frac{v^{2}}{C^{2} R}=0
$$

or

$$
S_{e}+S_{a}+S_{f}=0,
$$

where $S_{e}$ is the energy slope, $S_{a}$ is the acceleration slope, and $S_{f}$ is the friction slope. Here, Henderson (1966) made a careful distinction between $S_{e}$ and $S_{f}$ In the steady flow case, $S_{e}$ is the same as $S_{f}$ except opposite in sign. However, in the unsteady flow case, $S_{e}$ and $S_{f}$ are defined as

$$
S_{e}=\frac{\partial H}{\partial x}
$$

and

$$
S_{f}=\frac{v^{2}}{C^{2} R}
$$

where $S_{e}$ is not equal to $S_{f}$ Instead,

$$
S_{e}+S_{a}=-S_{f}
$$

where $S_{a}=\frac{1}{g} \frac{\partial v}{\partial t} . S_{a}$ is a source of energy loss or gain due to the unsteadiness of the flow.
Setting $h=z+y$, equation 24 can be rewritten

$$
\frac{\partial H}{\partial x}=\frac{\partial z}{\partial x}+\frac{\partial y}{\partial x}+\frac{v}{g} \frac{\partial v}{\partial x} .
$$

Substituting $S_{o}=\frac{\partial z}{\partial x}$

$$
\frac{\partial H}{\partial x}=-S_{o}+\frac{\partial y}{\partial x}+\frac{v}{g} \frac{\partial v}{\partial x} .
$$

Finally, substituting equation 35 into equation 29 and rearranging, a commonly seen form of the equation of motion is obtained:

$$
S_{f}=\frac{v^{2}}{C^{2} R}=S_{o}-\frac{\partial y}{\partial x}-\frac{v}{g} \frac{\partial v}{\partial x}-\frac{1}{g} \frac{\partial v}{\partial t} .
$$

\section{Significant}

terms for:

steady uniform

flow,

steady nonuniform flow,

unsteady nonuniform

flow.

The above arrangement was pointed out by Henderson (1966). It shows that additional terms must be evaluated as the complexity of a given water-routing problem increases. Equation 36 can be rearranged into another commonly seen form by multiplying by $g$ and using $S_{f}$ instead of the Chézy equation:

$$
g \frac{\partial y}{\partial x}+v \frac{\partial v}{\partial x}+\frac{\partial v}{\partial t}=g\left(S_{o}-S_{f}\right)
$$

Equation 37 is the one-dimensional form of the equation of motion describing unsteady open-channel flow with zero lateral inflow. Other more complex applications may require modification of certain terms of additional terms to describe situations such as nonprismatic channels, lateral inflow, and flood-plain storage. Twoor three-dimensional flow requires an entirely different development. According to Viessman and others (1977, p. 562), the convective terms $g \partial y / \partial x$ and $v \partial v / \partial x$ in equation 37 describe the changes in kinetic energy and potential energy (also called the inertia terms), the term $\partial v / \partial t$ accounts for the local acceleration of the fluid (also called the pressure-differential term), and 
the terms $g S_{o}$ and $g S_{f}$ account for the component of gravitational force in the direction of flow and friction along the channel. Equations 6 and 37 are sometimes called the Saint Venant equations.

\section{DEVELOPMENT OF KINEMATIC-WAVE APPROXIMATIONS}

Kinematic-wave approximations to equation 37 were first named and described in detail by Lighthill and Whitham (1955). Waves whose motion is adequately described by these approximations are known as "kinematic waves" as opposed to dynamic waves as described by equation 37 . The various equations and relationships describing kinematic waves constitute "kinematic-wave theory." When this theory is applied to channel-water routing or overland-flow routing, it is often called "kinematic-flood routing," "kinematic model," or "kinematic flow" (Miller and Cunge, 1975).

To apply kinematic-wave theory, the shallow-water wave is assumed to be long and flat so that the friction slope, $S_{f}$ is nearly equal to the bed slope, $S_{o}$, in equation 37.Thus, the remaining terms, $\frac{\partial v}{\partial t}, v \frac{\partial v}{\partial x}$, and $g \frac{\partial y}{\partial x}$, sometimes called the secondary terms, are assumed to be insignificant. This implies that there is a balance between the gravitational and frictional forces. The resulting equation becomes

$$
\mathrm{O}=g\left(S_{o}-S_{f}\right)
$$

or

$$
S_{o}=S_{f}
$$

From equation 39 it is obvious that the friction slope is parallel to the bed slope and, therefore, does not change with flow conditions. Thus, it follows that $q$ is a function of $y$ or $A$ alone and can be evaluated using any of the uniform-flow equations, such as the Chézy or Manning formulas, in which $S_{o}$ is substituted for $S_{f}$ The terms neglected in the development of equation 39 are the inertia and pressure-differential terms-the dynamic terms. Kinematics is usually defined as the description of motion without considering the forces giving rise to motion. The kinematic-wave model is so named because it is based primarily on the continuity equation and only approximates the dynamic equation with a uniform-flow formula.

By defining the friction slope with a uniform-flow formula, equation 39 can be represented for application to a specific channel or overland-flow plane by a general power relationship of the form

$$
Q=\alpha A^{m},
$$

in which $\alpha$ and $m$ are coefficients defined for each channel cross section. Methods for estimating $\alpha$ and $m$ are given under "Supplemental Information." Usually, in an application of kinematic-wave theory, equation $\mathbf{4 0}$ and the unsteady continuity equation (eq. 6) are solved simultaneously. Equations 6 and 40 are called the kinematic-wave equations.

Recalling the unsteady continuity equation (eq. 6), $\frac{\partial Q}{\partial x}+\frac{\partial A}{\partial t}=0$, and noting that the kinematic-wave celerity, $c$, is equal to $\frac{\partial Q}{\partial A}$ and that $\frac{\partial A}{\partial t}$ can be written $\frac{\partial A}{\partial Q} \frac{\partial Q}{\partial t}$, the wave celerity can be substituted into equation 6 to obtain what is often called the kinematicwave equation,

$$
\frac{1}{c} \frac{\partial Q}{\partial t}+\frac{\partial Q}{\partial x}=0
$$

\section{IMPORTANCE OF ASSUMPTIONS AND APPROXIMATIONS}

To avoid applying the kinematic-wave theory to situations that it does not adequately describe, the assumptions and approximations made to develop the theory need to be understood. Because the kinematicwave equations are based on the dynamic-wave equations, the assumptions and approximations made in the development of the latter need to be examined first. The details in the following descriptions, except as otherwise referenced, for dynamic waves are taken from Liggett (1975) and for kinematic waves are taken from Lighthill and Whitham (1955), Yen (1979), Miller and Cunge (1975), and Ponce and Simons (1977).

\section{DYNAMIC WAVES}

Slope and hydrostatic pressure approximations.-In equation 11 the pressure distribution is assumed to be hydrostatic. The pressure distribution will depart from hydrostatic when the bed slope becomes steep. Chow $(1959$, p. 32-33) showed that for uniform flow in steep channels the pressure at any depth must be corrected by the factor $\cos ^{2} \theta$. While this factor is only approximate for unsteady flows, it provides an indication of the magnitude of errors involved. The correction $\left(\cos ^{2} \theta\right)$ decreases the pressure by an amount less than 1 percent until $\theta$ is nearly 6 degrees, or a slope of about 1 in 10. Ordinary channels usually have slopes that are 
much less than 6 degrees. Therefore, this approximation commonly is insignificant.

For the pressure distribution to be hydrostatic, it is also necessary that the vertical components of acceleration be negligible. This is true if the flow is not rapidly varied and for long shallow-water waves where the slope of the wave front or back is small. However, this may not be true for shorter, deeper water waves. As the waves approach oscillatory waves, a correction should be made to the wave celerity (wave speed). In order to maintain a 2-percent accuracy in the wave celerity, the ratio of the depth of the wave to the length of the wave must be less than or equal to 0.055 (Liggett, 1975, p. 44). Because the depth of a flood wave is usually less than a few tens of feet and the length is usually measured in miles, the ratio is much less than 0.055 and this approximation is commonly insignificant.

Friction approximations.-Two friction-slope approximations are described in the section "Supplemental Information" under "Equation Development Concepts." The first approximation is the common use of the Chézy and Manning formulas to estimate the friction slope in unsteady flow even though they were developed for steady uniform flow. Results of unsteady flow modeling indicate that they are applicable. One major problem is how to estimate the resistance or friction coefficient. An error in the estimation of the resistance coefficient leads to an error in the predicted velocity or flow of the same magnitude. The second approximation is the assumption of steady flow made to exprss the longitudinal shear stress using the Chézy formula. Again, the results of unsteady flow modeling indicate that this approximation is adequate.

\section{KINEMATIC WAVES}

The additional assumptions and approximations made in kinematic-wave theory can be summarized by noting that $Q$ is assumed to be a function of $y$ alone. This means that $S_{f}=S_{o}$ and the other three slope terms. called the secondary terms, in equation 36 are negligible. Thus, the bed slope, $S_{o}$, is assumed to be large enough and the water wave long and flat enough so that the change in depth and velocity with respect to distance $\left(\frac{\partial y}{\partial x}\right.$ and $\left.\frac{\partial v}{\partial x}\right)$ and the change in velocity with respect to time $\left(\frac{\partial v}{\partial t}\right)$ are negligible when subtracted from $S_{o}$ in equation 36 .

Henderson (1966, p. 364) pointed out that for natural floods in steep rivers whose slopes are of the order of 10 feet per mile or more, the values of the secondary terms are small. He also provides some typical values for the slope terms taken from an actual river in steep alluvial country as follows:

$$
\begin{array}{cccc}
\text { So, } & \frac{\partial y}{\partial x}, & \frac{v}{g} \frac{\partial v}{\partial x}, & \frac{1}{g} \frac{\partial v}{\partial t} \\
26 & 0.05 \quad \begin{array}{c}
0.125-0.25 \\
\text { (feet per mile) }
\end{array} & 0.05
\end{array}
$$

Gunaratnam and Perkins (1970, p. 45) also reported the following values, taken from Schaake (1965), for gutter and overland flow.

$$
\begin{array}{cccl}
\text { So, } & \frac{\partial y}{\partial x}, & \frac{v}{g} \frac{\partial v}{\partial x}, & \frac{1}{g} \frac{\partial v}{\partial t} \\
182 & 9.8 & 4.9 & 4.9 \\
212 & \begin{array}{l}
16.4 \\
\text { (feet per mile) }
\end{array} & 1.64
\end{array}
$$

Values for the other slope terms (secondary terms) are small compared to $S_{o}$, which indicate the rationsle behind the assumptions made in the development of kinematic-wave theory.

\section{PROPERTIES OF KINEMATIC WAVES}

The properties or behavior of kinematic waves were first described in detail by Lighthill and Whitham (1955). They were described further by Henderson (1963 and 1966).

Unlike dynamic waves which propagate both downstream and upstream at characteristic speeds as stated in the equation, $c=v \pm \sqrt{g y}$ (positive downstream and negative upstream); kinematic waves propagate downstream only. Lighthill and Whitham (1955, p. 282) pointed out that the continuity equation (eq 6) is of the first order because for kinematic waves $Q$ is a function of $y$ alone; thus, kinematic waves have only one wave speed.

According to Henderson (1966, p. 365-367), the wave speed in a prismatic channel with no lateral inflow can be determined as follows. If a monoclinal wave is brought to rest by the superposition of a velocity equal and opposite to the wave velocity (or celerity), $c$, the discharge through the now stationary wave is constant and equal to

$$
(v-c) A
$$

or $Q-c A=$ constant discharge through wave. Taking the derivative, 


$$
\mathrm{d} Q-c \mathrm{~d} A=0,
$$

and rearranging,

$$
c=\frac{\mathrm{d} Q}{\mathrm{~d} A} \text {. }
$$

This is called the Kleitz-Seddon law (Lighthill and Whitham, 1955, p. 282). Another form may be obtained by noting that $\mathrm{d} A=B \mathrm{~d} y$ :

$$
c=\frac{1}{B} \frac{\mathrm{d} Q}{\mathrm{~d} y}
$$

Setting $Q=A v$ in equation 44 ,

$$
c=\frac{\mathrm{d}(A v)}{\mathrm{d} A}=v \frac{\mathrm{d} A}{\mathrm{~d} A}+A \frac{\mathrm{d} v}{\mathrm{~d} A}
$$

or

$$
c=v+A \frac{\mathrm{d} v}{\mathrm{~d} A} .
$$

The wave celerity can be evaluated further for wide rectangular channels when using a specific uniformflow equation such as the Chézy or Manning formulas. For example, using the Chezy formula to describe the relationship between velocity and depth and substituting $R=A / P$, the following form of the equation is obtained:

$$
v=C \sqrt{\frac{A}{p} S_{o}}=C P^{-1 / 2} S_{o}^{1 / 2} A^{1 / 2}
$$

Taking the derivative with respect to $A$ and noting that for wide rectangular channels $P \cong B$ and is approximately constant,

$$
\frac{\mathrm{d} v}{\mathrm{~d} A}=1 / 2 C P^{-1 / 2} S_{o}^{1 / 2} A^{-1 / 2} ;
$$

multiplying both sides by $A$,

$$
\mathrm{A} \frac{\mathrm{d} v}{\mathrm{~d} A}=1 / 2 C P^{-1 / 2} S_{o}^{1 / 2} A^{1 / 2}=1 / 2 C \sqrt{\frac{A}{p} S_{o}} ;
$$

substituting equation 48 into 50 ,

$$
A \frac{\mathrm{d} v}{\mathrm{~d} A}=1 / 2 v
$$

and, substituting equation 51 into equation 47 ,

$$
c=v+1 / 2 v=3 / 2 v \text {. }
$$

When the Manning formula is used, equation 52 by a similar procedure becomes

$$
c=5 / 3 v \text {. }
$$

The relationship between the wave celerity and mean velocity can be evaluated when the characteristics of specific channels are defined using equation 40 . From equation 44 it can be seen that the wave celerity can be determined if equation $\mathbf{4 0}$ is differentiated with respect to $A$ as follows:

$$
c=\frac{\mathrm{d} Q}{\mathrm{~d} A}=\alpha m A^{m-1}=\frac{\alpha m}{A} A^{m} .
$$

Substituting equation 40 ,

$$
c=m \frac{\mathbf{Q}}{\mathbf{A}}=m v .
$$

Therefore, $m$ determines the relationship between the wave celerity and the mean velocity. Table 1 in the "Supplemental Information" section shows that for some typical prismatic channels the wave celerity is 1 to 1.67 times as fast as the mean velocity.

On streams where stage-discharge ratings have been developed at representative channel sections, the kinematic-wave celerity can be estimated using equation 45 . The slope $\frac{\mathrm{d} Q}{\mathrm{~d} y}$ can be measured directly from the rating curve. This often provides a useful approximation of wave celerities in natural channels.

Equation 44 gives the speed at which a point of constant $Q$ and $y$ is moving. Since this speed depends on $y$ alone, points on the wave profile at the same depth, for example $B$ and $B^{\prime}$ at time, $t$, in figure 4 , move at the same velocity, and at a later time, $t+\Delta t$, the chord length $B-B^{\prime}$ remains constant. Thus, the wave does not lengthen or disperse and does not subside as it moves downstream (see point $A$ in fig. 4). However, it does distort because $\mathrm{d} Q / \mathrm{d} y$, and thus $v$, increases with $y$. This was described by Henderson (1963 and 1966, p. 370). If the wave traveled far enough, point $A$ would overtake point $B^{\prime}$ forming a vertical wall of water, or surge. This result of kinematic-wave theory was named "kinematic shock" by Lighthill and Whitham (1955). Before kinematic shock can be discussed, the different solution techniques for the kinematic-wave equations need to be introduced.

\section{SOLUTION TECHNIQUES}

The equations describing dynamic or kinematic waves are partial-differential equations for which 


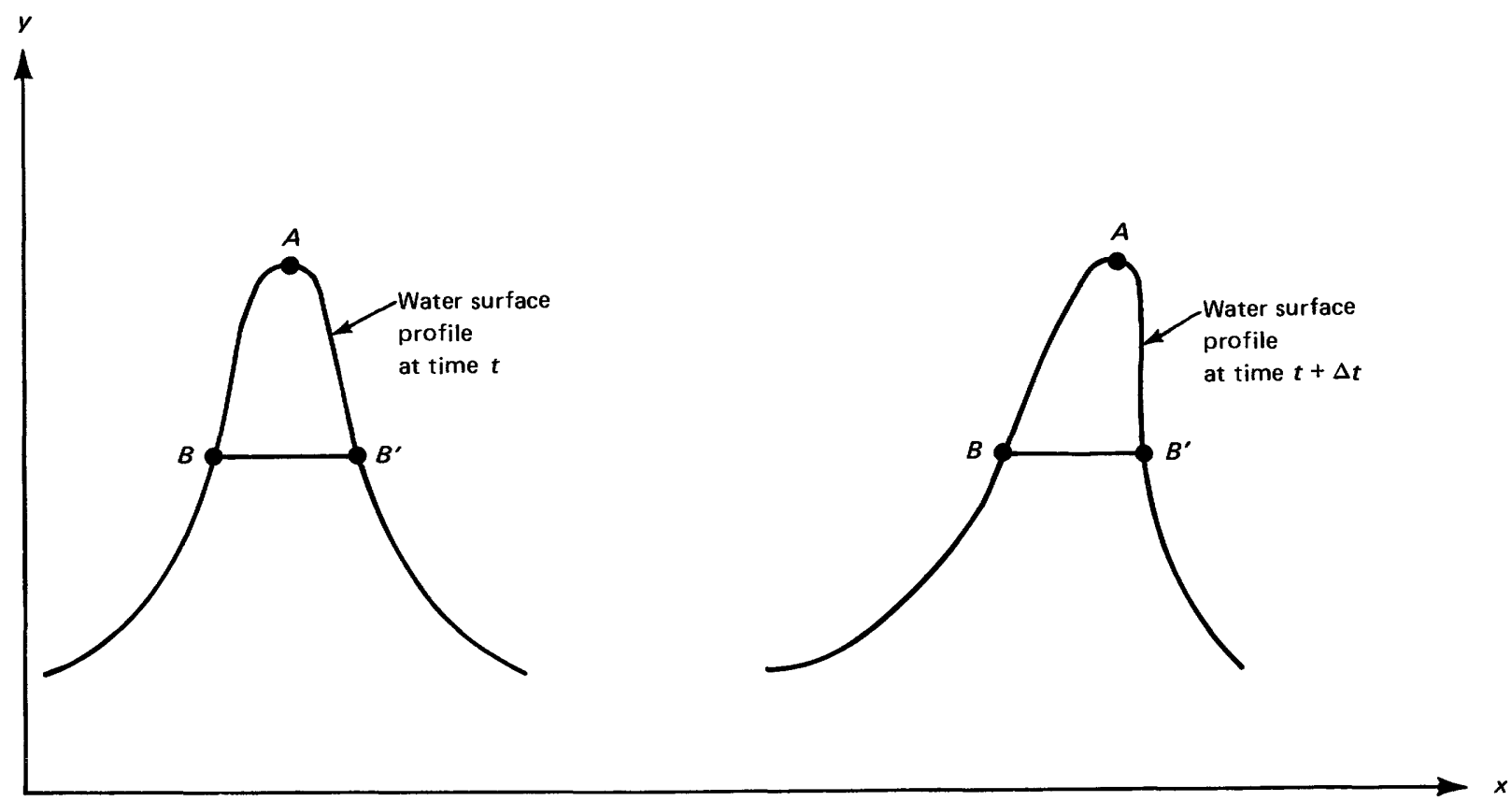

FIGURE 4.-Water-surface profiles at time, $t$ and $t+\Delta t$, showing distortion or steepening of the kinematic wave.

there are no analytic solutions except in a few idealized situations. Thus, numerical techniques such as finitedifference methods, the method of characteristics, or finite-element methods are employed. The first two are most commonly used and will be discussed here. The details involved in applying these techniques are described by Liggett and Cunge (1975). The application of numerical solution techniques to partial-differential equations is a complex topic. While a complete description of the concepts involved is not included in this report, an overview of the basic concepts is given because the techniques are required to use kinematicwave models.

Partial-differential equations, in general, can be classified as elliptic, parabolic, or hyperbolic. Equilibrium or steady-state problems generally are described by equations that are elliptic. Propagation for marching problems are described by parabolic and hyperbolic equations. The equations can be classified using the concept of characteristics. Elliptic equations have no real characteristic directions, parabolic equations have one characteristic direction, and hyperbolic equations have two real characteristic directions. The details involved in classifying partial-differential equations are given by Ames (1977, p. 3-9). The equations describing kinematic waves behave as parabolic equations although, since they are not of the second order they are not actually parabolic. The equations describing dynamic waves are hyperbolic. It has been noted previously that kinematic waves propagate in only one characteristic direction while dynamic waves propagate in two characteristic directions. The difference in the number of propagation directions is an example of how the solutions to the equations in different classifications can behave differently. This needs to be considered in the formulation of the solutions.

\section{FINITE-DIFFERENCE METHODS}

The basic concepts behind finite-difference approximations to partial-differential equations are given by Ames (1977). The approximations can be developed in the following manner. If, in the $x-y$ plane, a Taylor series for an arbitrary point $u(x+\Delta x, y)$ is developed about $u(x, y)$, the approximation for $\partial u / \partial x$ can be developed.

$$
\begin{aligned}
u(x+\Delta x, y)= & u(x, y)+\frac{\Delta x}{1 !} \frac{\partial u(x, y)}{\partial x} \\
& +\frac{(\Delta x)^{2}}{2 !} \frac{\partial^{2} u(x, y)}{\partial x^{2}} \\
& +\ldots \frac{(\Delta x)^{(n-1)}}{(n-1) !} \frac{\partial^{(n-1)} u(x, y)}{\partial x^{(n-1)}} \\
& +O\left[|\Delta x|^{n}\right],
\end{aligned}
$$

where $O\left[|\Delta x|^{n}\right]$ is read "terms on the order of $\Delta x$ to the nth power." This is the notation for the truncation er- 
ror of this approximation. Simplifying by neglecting all terms of higher order than one and solving for $\partial u / \partial x$.

$$
\frac{\partial u(x, y)}{\partial x}=\frac{u(x+\Delta x, y)-u(x, y)}{\Delta x}+\frac{\mathrm{O}\left[|\Delta x|^{2}\right]}{\Delta x}
$$

or

$$
\frac{\partial u}{\partial x} i_{y}=\frac{u_{i+1, j}-u_{i, j}}{\Delta x}+\mathrm{O}[|\Delta x|]
$$

where the notation used in equation 58 is typical for approximation of $\partial u / \partial x$ in space and time for marching problems on a fixed grid in the $x-t$ plane. Points on the grid are located by multiplying the grid spacing, $\Delta x$ or $\Delta t$, by the corresponding $i$ or $j$ terms.

Equation 58 is a one-dimensional forward difference. The same procedure can be repeated for $u(x-\Delta x, y)$ to determine a backward-difference approximation. If the backward and forward differences are written to include the second order terms and then one is subtracted from the other, a central difference of the second order, $\mathrm{O}\left[|\Delta x|^{2}\right]$, is obtained. Finally, if the same procedures are followed except that the two differences are added, a second order approximation for $\partial^{2} u / \partial x^{2}$ is obtained. Following the above procedures, approximations for the terms in the partial-differential equations describing one-dimensional unsteady flow can be developed.

To apply finite-difference techniques, a fixed grid in the $x-t$ plane is usually employed as shown in figure 5 . Generally, in unsteady flow problems such as river routing, the velocity and depth $(v, y)$ or discharge and area $(Q, A)$ are known for all grid points along the $x$-direction at the initial time, $t=0$. Also, the inflow hydrograph is known (either $y$ or $Q$ ) and sometimes the outflow hydrograph is known. To solve for the points between, either an explicit or implicit scheme is em-

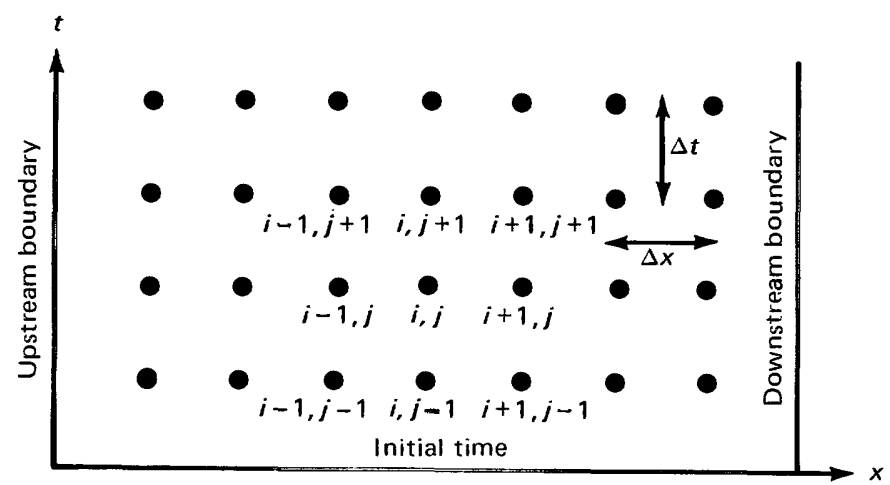

Figure 5.-Fixed grid in the $x-t$ plane showing location of grid points and counters, $i$ and $j$. ployed. Generally, when an explicit scheme is used, the approximations are written in such a way that unknowns at each point in the next time step can be solved for individually and then the procedure is repeated for each succeeding row.

Explicit schemes are often subject to a certain condition that is expressed as a "mesh ratio" (ratio of time step to distant step size) to produce a stable finitedifference scheme (O'Brien and others, 1950). Stability refers to the ability of the scheme to prevent numerical errors from growing in an unbounded or uncontrolled manner. This stability condition is called the Courant or Courant-Friedrichs-Lewy stability condition. It states, for application to the equations in this report, that the wave celerity must be less than the ratio of distance step to time step in the finite-difference scheme. In practical applications, this condition can limit the size of time step that can be used.

Generally, when an implicit scheme is used, unknowns for more than one point or each succeeding row of points in time are solved for at once. Finitedifference equations involving more than one unknown point in the unknown time step are developed so that each grid point along the $x$-direction is included. The scheme is designed so that, when the equations are written for an entire row, there are an equal number of unknowns and equations. Then a Gaussian elimination or other matrix-solver is used to solve for unknowns at all points simultaneously. One of the advantages of implicit schemes over explicit schemes is that they are generally not subject to the restrictive mesh ratio conditions for stability.

To apply the finite-difference methods, care must be taken to describe the initial and boundary conditions properly. In the application of a kinematic-wave model all of the many different hydrographs and ranges of flow are described by the same partial-differential equations (eqs. 6 and 40). The many possible flows are distinguished only by the initial and boundary conditions and by the channel parameters, such as the friction coefficient and channel shapes. In the application of a kinematic-wave model, initial values for the unknowns are required at all grid locations and values for the unknowns are required at the upstream boundary of the channel for all time steps modeled. The actual unknowns specified depend on the formulation of the model but are usually some combination of velocity, depth, discharge, or area. Only one is specified for each initial or boundary condition. The application of a dynamic-wave model requires the specification of more complex boundary conditions because the equations have two characteristic directions. Therefore, initial conditions and both upstream and downstream boundary conditions must be specified. 
Finite-difference approximations for the kinematicwave equations have been presented by Brankensiek (1967), $\mathrm{Li}$ and others (1975), $\mathrm{Li}$ and others (1976), Dawdy and others (1978), and Kibler and Woolhiser (1970). As an example, the explicit scheme by Dawdy and others (1978) is described under "Supplemental Information." A linear, implicit finite-difference solution for the full dynamic-wave equations is presented by Land (1978) and Keefer (1976).

It should be noted that the application of numerical methods to partial-differential equations is not straightforward. Finite-difference schemes are often developed that appear to be accurate and yet do not produce useful results. However, a very similar scheme may produce very good results (Roache, 1976, p. 3).

\section{METHOD OF CHARACTERISTICS}

The method of characteristics has been used to solve the equations describing dynamic and kinematic waves. An overview of the development of the method of characteristics for application to the kinematic-wave equations is presented here. The method applied to the full dynamic-wave equations is described by Abbott (1975) and Theurer (1975). The development given here is taken from Eagleson (1970) and Borah and others (1980). A detailed discussion of the concepts behind the method of characteristics is given by Crandall (1956, $p$. 352-355). The method involves locating the characteristic paths and then integrating the ordinary differential equations that apply along the characteristic paths. When applied to wave motions, the method describes the space-time locus of discontinuity in the partial derivatives, with respect to space and time, of the dependent variables (in this case, $A$ and $Q$ ). This locus defines the path of wave propagation (the characteristic path) along which the phenomenon is described by an integrable ordinary differential equation.

The development of the method of characteristics for the kinematic-wave equations involves a system of four equations in four unknowns written in matrix form. The four unknowns are the partial derivatives, with respect to space and time, of the dependent variables, $\frac{\partial A}{\partial t}, \frac{\partial A}{\partial x}, \frac{\partial Q}{\partial t}$, and $\frac{\partial Q}{\partial x}$. After the system of equations is written in matrix form, the remaining concepts in the development are based on Cramer's rule for solving simultaneous linear equations. Cramer's rule gives the solution in the form of quotients of determinants.

The application of the method of characteristics to kinematic-wave equations converts the two equations, one of which is a partial-differential equation, to ordinary differential equations. One describes the path of the characteristic in the $x-t$ plane, and the other describes the changes in area or discharge along the characteristic path. The path of the characteristic is determined by the speed of the kinematic wave (wave celerity). This speed is dependent on depth only; thus, for prismatic channels in which there is no lateral inflow, the speed is constant and the characteristic is a straight line (fig. 6). For nonprismatic channels or situations in which there is lateral inflow, the speed varies due to the varying depth and the characteristic is not a straight line. As pointed out earlier, the kinematic wave does not disperse or attenuate. Therefore, for the simple case of no lateral inflow, the method of characteristics involves simply tracking point discharges or areas (sometimes velocity and depth are used) to downstream locations based on the wave celerity. The method is developed as follows.

When applying kinematic-wave theory to overland flow, where lateral inflow must be considered, the continuity equation (eq. 6) is written to include lateral inflow:

$$
\frac{\partial Q}{\partial x}+\frac{\partial A}{\partial t}=q
$$

where $q$ is lateral inflow in the form of either rainfall excess or overland flow to a receiving channel. Following the simplifications made in the kinematic-wave theory, the equation of motion (eq. 40) is written, based on a uniform-flow equation, as $Q=\alpha A^{m}$. $\partial Q / \partial t$ can be determined from equation 40 :

$$
\frac{\partial Q}{\partial t}=\alpha m A^{m-1} \frac{\partial A}{\partial t}
$$

In addition to equations 59 and 60 , two more equations can be added using the definition of a total differential:

$$
\mathrm{d} Q=\frac{\partial Q}{\partial x} \mathrm{~d} x+\frac{\partial Q}{\partial t} \mathrm{~d} t
$$

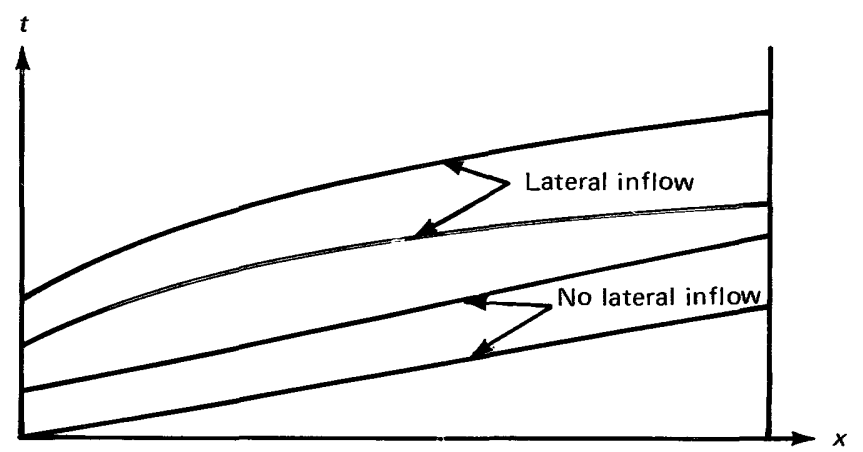

FIgURE 6.-Schematic of $x-t$ plane showing typical characteristic paths for the kinematic-wave equations, with and without lateral inflow. 
and

$$
\mathrm{d} A=\frac{\partial A}{\partial x} \mathrm{~d} x+\frac{\partial A}{\partial t} \mathrm{~d} t
$$

Equations 59, 60, 61, and 62 can be written in matrix form:

$$
\left|\begin{array}{crrr}
1 & 0 & 0 & 1 \\
\alpha m \cdot A^{m-1} & 0 & -1 & 0 \\
\mathrm{~d} t & \mathrm{~d} x & 0 & 0 \\
0 & 0 & \mathrm{~d} t & \mathrm{~d} x
\end{array}\right|\left|\begin{array}{c}
\partial A / \partial t \\
\partial A / \partial x \\
\partial Q / \partial t \\
\partial Q / \partial x
\end{array}\right|=\left|\begin{array}{r}
q \\
0 \\
\mathrm{~d} A \\
\mathrm{~d} Q
\end{array}\right|
$$

The discontinuity in the four derivatives (or the path of the characteristic line) is determined by the indeterminacy in their solution. The derivatives in equation 63 do not exist along the characteristic paths and, therefore, there is no unique solution to 63 along the characteristic paths. According to Cramer's rule, a solution to the four equations exists and is unique unless the determinant

$$
\left|\begin{array}{crrr}
1 & 0 & 0 & 1 \\
\alpha m A^{m-1} & 0 & -1 & 0 \\
\mathrm{~d} t & \mathrm{~d} x & 0 & 0 \\
0 & 0 & \mathrm{~d} t & \mathrm{~d} x
\end{array}\right|=0
$$

or the ordinary differential equation

$$
\left(\frac{\mathrm{d} x}{\mathrm{~d} t}\right)^{2}-\alpha m A^{m-1} \frac{\mathrm{d} x}{\mathrm{~d} t}=0 .
$$

Equation 65 has two roots for the wave speed $=\mathrm{d} x / \mathrm{d} t=c$ :

$$
c=\alpha m A^{m-1}
$$

and

$$
c=0 \text {. }
$$

Except for the conditions of infinite roughness-zero slope and zero depth-the root in equation 66 is trivial. Thus, equation 54 is used to describe the kinematicwave characteristic. As shown earlier, equation 54 can be rewritten

$$
c=m v
$$

Since $m$ in the approximation to the uniform-flow equation is usually larger than 1 , it can be seen from equation 55 that the wave speed is greater than the stream velocity and propagates in the positive or downstream direction only. According to Cramer's rule, when equation 55 holds, there is no solution at all unless the other determinants in equation 63 also equal zero. Now, if the columns of the coefficient matrix are replaced one at a time by the column vector on the right-hand side of equation 63 , the determinant set equal to zero, and solved, the following are obtained. Replacing the first column or third column

$$
\frac{\mathrm{d} Q}{\mathrm{~d} x}=q ;
$$

replacing the second column,

$$
\frac{\mathrm{d} A}{\mathrm{~d} t}=q
$$

and, replacing the fourth column,

$$
\frac{\mathrm{d} Q}{\mathrm{~d} t}=q \alpha m A^{m-1}
$$

Dividing equation 68 by equation 54 ,

$$
\frac{\mathrm{d} A}{\mathrm{~d} x}=\frac{q}{\alpha m A^{m-1}} .
$$

Equations 67 through 70 apply only along the characteristic path defined by equation 55 .

Borah and others (1980) integrated and discretized these equations assuming that $q$ remains constant over small time and space increments. The solution is nearly analytic and involves very little numerical dispersion. However, the discretization does introduce some approximations. The characteristic equations are solved conveniently by setting $\Delta x$ or $\Delta t$ and then computing the corresponding $\Delta t$ or $\Delta x$ value. When lateral inflow is included, the characteristic equations are

$$
\Delta x_{i}=\frac{\alpha}{q_{j}}\left[\left(q_{j} \Delta t_{j}+A_{i-1, j-1}\right)^{m}-\left(A_{i-1, j-1}\right)^{m}\right]
$$

or

$$
\Delta t_{j}=\frac{1}{q_{j}}\left\{\left[\frac{q_{j}}{\alpha} \Delta x_{i}+\left(A_{i-1, j-1}\right)^{m}\right]^{1 / m} A_{i-1, j-1}\right\}
$$

When lateral inflow is not included, equations 71 and 72 are not defined. The characteristic equations for zero lateral inflow are

$$
\Delta x_{i}=\alpha m\left(A_{i-1, j-1}\right)^{m-1} \Delta t_{j}
$$

or

$$
\Delta t_{j}=\frac{1}{\alpha m}\left(A_{i-1, j-1}\right)^{1-m} \Delta x_{i}
$$


The equations for area or discharge along the characteristics are

$$
A_{i j}=A_{i-1, j-1}+q_{j} \Delta t_{j}
$$

or

$$
Q_{i, j}=Q_{i-1, j-1}+q_{j} \Delta x_{i}
$$

One possible procedure for solving the above equations is to determine the downstream location to which an upstream hydrograph is to be routed (define $\Delta x$ ). From the known $\Delta x, \Delta t$ can be computed from equation 72 or 74 , the cross-sectional area of the flow at the new location $(x+\Delta x, t+\Delta t)$ can be computed from equation 75 , and the discharge can be computed using equation 40 . To follow this procedure, the upstream hydrograph must be known at each increment of time at the initial distance $x$. Then the hydrograph can be computed at the downstream distance $x+\Delta x$.

\section{KINEMATIC SHOCK}

When the kinematic-wave equations are solved using the method of characteristics (where numerical dispersion is not a problem), kinematic shocks do occur. Some investigators have attempted to determine whether these shocks occur physically. However, Henderson (1966, p. 370) pointed out that while the steepening of the wave front may suggest that a surge will form, as the wave front becomes steeper the neglected secondary slope terms in equation 37 will become significant. The effect of these terms is to introduce dispersion and attenuation that will retard the steepening of the wave front, probably delaying and preventing any formation of a surge. Henderson's point indicates that shocks are simply a result of the approximations made in the development of the theory.

In the development of the kinematic-wave equations, the secondary slope terms in equation 37 are assumed to be small enough to be neglected when the bed slope is large. However, even though these terms are small, the effect of neglecting them is cumulative. In figure 4, the steepening of the wave front is small because of the small differences in celerity at each depth in the kinematic wave. However, the steepening is continuous because there are no terms in the kinematic-wave model to prevent it. The peak always has a greater depth and, therefore, a faster mean velocity and wave celerity than a lower point on the front face of the hydrograph. Thus, the peak continuously overtakes the lower depths of the hydrograph and shock occurs, making the solution unreasonable. The inclusion of the secondary slope terms in the equation of motion would introduce a small amount of dispersion and attenuation that would tend to offset the tendency to steepen. This would slow the steepening of the wave front continuously, although by a very small amount. Again, if the wave front eventually does become steeper, the secondary slope terms become even more significant and will probably prevent the formation of a surge. After any significant travel distance, the kinematicwave approximation will always tend to steepen the routed wave more than predicted by the full dynamic equations due to the cumulative effect of neglecting the secondary terms in the equation of motion.

Kibler and Woolhiser (1970) and Li and others (1976) describe the conditions that cause kinematic shock formation based on the paths of the characteristics of the kinematic-wave equations. The equation describing the path of the characteristics is $\mathrm{d} x / \mathrm{d} t=c$. This is the path of wave propagation-the path that an observer would follow if he was moving with the wave. When the channel shape remains constant and there is no lateral inflow, the wave celerity, $c$, is a constant for a specific depth. Therefore, the characteristic paths are straight lines. The slope of the lines is dependent on the depth of the flow. Water-surface profiles at two times and the characteristic paths between them are plotted in figure 7 , showing the condition necessary for shock formation (adapted from Peter E. Smith, written commun., 1981). This condition is that the characteristic paths must cross. The characteristic paths cross because a deeper and, thus, faster element of the flood wave overtakes a shallower and, thus, slower element of the wave. This causes a severe steepening of the wave front and results in kinematic shock.

The condition for an intersection of the characteristic paths is that the slope, $\mathrm{d} x / \mathrm{d} t$, of the upper characteristic, such as path 2 in figure 7 , must be greater than that of the lower characteristic, such as path 1 in figure 7. This condition can occur with or without lateral inflow. Following Kibler and Woolhiser (1970, p. 8), this condition can be described as

$$
h_{2}-h_{1}>q\left(t_{2}-t_{1}\right)
$$

where

$$
\begin{aligned}
h_{1} \text { and } h_{2}= & \text { height of water above datum at a particu- } \\
& \text { lar point corresponding to a time, } t_{1} \text {, and } \\
& \text { a later time, } t_{2} \text {, }
\end{aligned}
$$

and

$$
q=\text { constant lateral inflow. }
$$




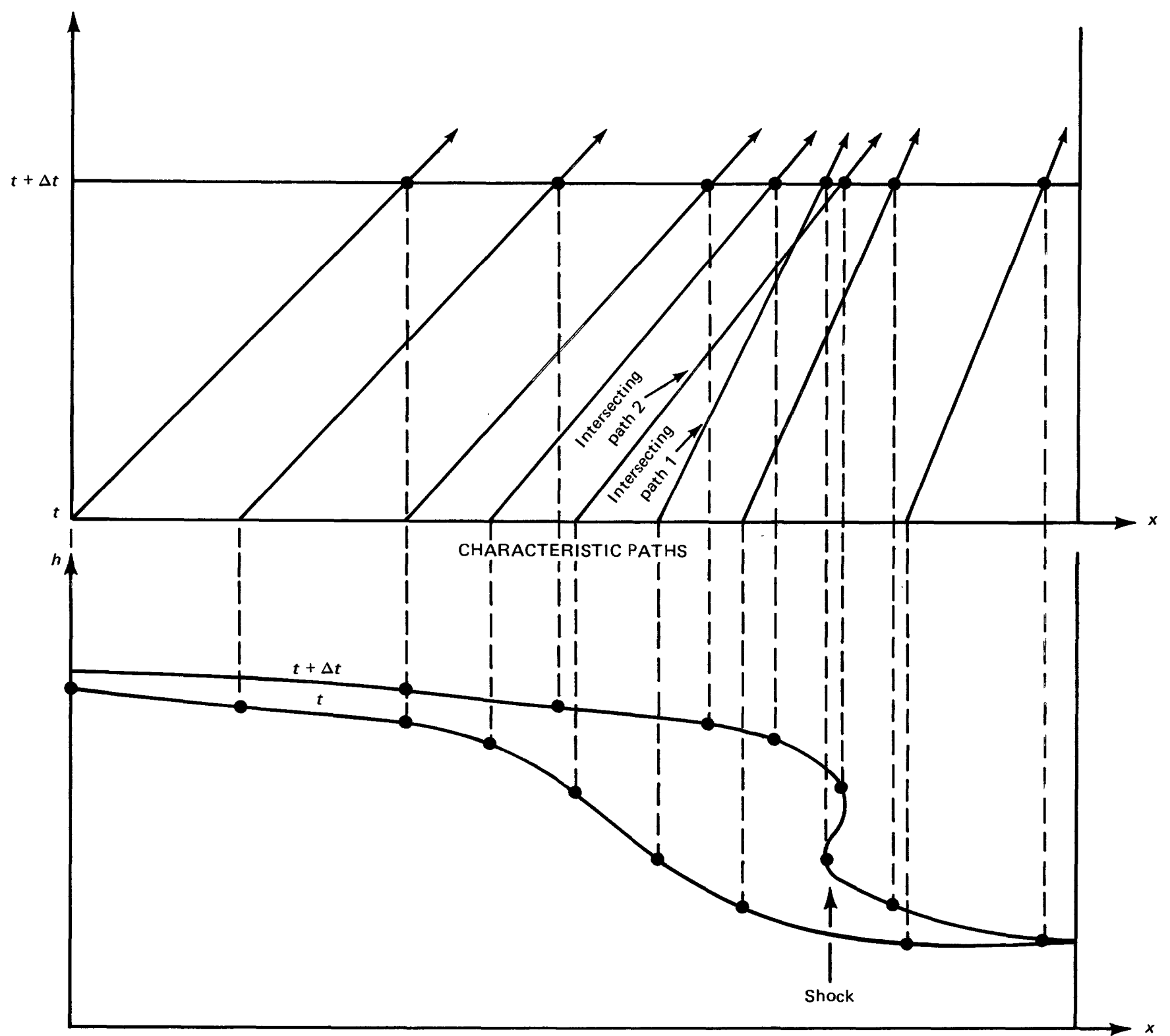

WATER SURFACE PROFILES

FIGURE 7.-Water-surface profiles at a time, $t$ and $t+\Delta t$, and the characteristic paths between them showing the condition necessary for shock formation.

An illustration of the cumulative effect of neglecting the secondary slope terms in the kinematic-wave approximation is provided Ponce, $\mathbf{L i}$, and Simons (1978). These authors developed a criterion that determines when the kinematic-wave model provides an adequate approximation of the diffusion- and dynamic-wave models. If the criterion is meet, the kinematic-wave model will provide 95-percent accuracy in the wave amplitude after one propagation period. Thus, for the usual situation in which the wave amplitude is being attenuated, the amplitude predicted by the diffusionor dynamic-wave models would be at least 95 percent of the amplitude predicted by the kinematic-wave model.
However, this is for one propagation period. After two propagation periods, the amplitude would be in error by 5 percent of a wave that was already in error by 5 percent. This criterion again indicates that the error is cumulative.

Usually, kinematic shocks only occur when the method of characteristics is used to solve the kinematic-wave equations (eqs. 6 and 40 ) because, in this case, the characteristics solution is a nearly analytic solution that, therefore, involves very little numerical dispersion. When numerical solutions such as finite-difference methods are used, the solution often includes a significant amount of numerical 
dispersion. This numerical dispersion has an effect on the solution similar to the secondary slope terms in equation 37. That is, it tends to introduce attenuation and dispersion. Numerical dispersion generally prevents the formation of kinematic shocks. While numerical dispersion is similar to the actual dispersion occurring in water waves, there is no control over how much dispersion and attenuation occurs. Therefore, it does not provide an accurate model unless the dispersion is controlled.

\section{DYNAMIC VERSUS KINEMATIC WAVES}

To mathematically describe dynamic waves requires both the continuity equation and the full equation of motion, whereas to describe kinematic waves requires the continuity equation and only a simplified form of the equation of motion. Dynamic waves propagate at two-wave celerities whereas kinematic waves propagate at one-wave celerity. According to Lighthill and Whitham (1955), the properties of both waves are demonstrated in flood waves but the kinematic properties of the waves dominate. This is indicated by the observed fact that the main part of the flood wave moves more slowly than the speed predicted for dynamic waves. Dynamic waves have a high rate of attenuation; therefore, the faster wave moving down the river ahead of the kinematic wave attenuates quickly and is, therefore, less significant a short distance downstream. Miller and Cunge $(1975$, p. 196) stated that the largest part of the flood wave moves as a kinematic wave because of the domination of the bedslope term in the equation of motion. Therefore, the speed of a flood wave may often be reliably predicted by the speed of a kinematic wave for wide channels, $c=3 / 2 v$, or from equation 45 .

\section{APPROXIMATIONS OF DYNAMIC WAVES}

The kinematic-wave approximation is only one of a number of possible approximations of dynamic waves. Ponce and Simons (1977) described a number of approximations by considering the various possible combinations of the slope terms included in the equation of motion (eq. 37) to be solved along with the continuity equation (eq. 6). They write equation 37 and number each term in the following form:

Term

Equation of motion
The wave models and terms included in each are as follows:
Model
1. Kinematic wave
2. Diffusion wave
3. Steady dynamic wave
4. Dynamic wave
5. Gravity

The terms are named as follows: $I$ is the local inertia term, II is the convective inertia term, III is the pressure-differential term, and IV is simply the bed and friction slopes. Ponce and Simons (1977) provided a detailed description of the properties of the five approximations based on their application of the theory of linear stability for flow in prismatic wide channels. A brief review of the properties of the diffusion-, steady dynamic-, and gravity-wave models follows (properties of the kinematic-wave approximation and the dynamicwave model were described previously).

Diffusion waves and kinematic waves propagate downstream only and at an equal celerity. However, unlike kinematic waves, diffusion waves attenuate as they propagate downstream. Steady dynamic waves also propagate downstream only and attenuate. However, the wave celerity and attenuation are not the same as for diffusion waves. Like dynamic waves, gravity wave propagate at two wave celerities, $c=v+\sqrt{g y}$ and $c=v-\sqrt{g y}$. However, unlike dynamic waves, they generally attenuate only weakly.

There are two other approximations that are of interest. Both are based on the diffusion-wave model. These approximations are the Muskingum-Cunge model and the diffusion-analogy model.

$$
\frac{\partial y}{\partial t}+c \frac{\partial y}{\partial x}=\mu \frac{\partial^{2} y}{\partial x^{2}}
$$

in which $\mu=$ diffusion coefficient.

In the diffusion-analogy model, the term $\mu \frac{\partial^{2} y}{\partial x^{2}}$ is used to approximate the diffusion introduced by the $\frac{\partial y}{\partial x}$ term in the diffusion-wave model. The diffusion-analogy model is developed under "Suplemental Information." The Muskingum-Cunge model is an adaptation of the Muskingum method in which the numerical dispersion is controlled based on the hydraulic characteristics of the channel. The Muskingum-Cunge model is described under "Special Topics and Applications."

The standard-step method commonly applied in stepbackwater analysis for steady, gradually varied flow is also an approximation of the dynamic-wave model. The 
development of the step-backwater approximation is given under "Supplemental Information."

Yen (1979) compared kinematic-, diffusion-, and steady dynamic-wave approximations to dynamic waves. He stated that the kinematic model is the least accurate and, even though the steady dynamic wave includes another term over the diffusion wave, it is not as accurate as the latter. This, he point out, is because for the typical case of downstream propagation terms I and II are usually of about the same order of magnitude but have opposite signs. Thus, neglecting both of them is more accurate than including only one.

When balancing the computation time and complexity against the accuracy required for each water-routing application, the kinematic-wave, the diffusion-wave or analogy, and the dynamic-wave models appear to be most commonly used. The remaining approximations usually entail almost as much complexity in their solutions and less accuracy when compared to the next more accurate model. Thus, the more accurate model is chosen.

\section{CRITERIA DESGRIBING LIMITATIONS OF APPLICABILITY OF APPROXIMATIONS}

Criteria for determining when kinematic-wave approximations of dynamic waves are acceptable have been examined by a number of authors. Miller and Cunge $(1975$, p. 197) showed the development of one criterion based on the Froude number (F). Setting dynamic- and kinematic-wave celerities equal produces a criterion for the condition of flow at which dynamic and kinematic waves are of equal importance. When the Froude number is equal to 2, the dynamic and kinematic waves have the same celerity. According to Miller and Cunge (1975), when $F$ is less than 2, dynamic waves are rapidly dampened out ( $F$ is less than 3/2 when Manning's equation is used). Kinematicwave theory does not adequately describe highly supercritical flows because the dynamic-wave celerity diverges from the kinematic-wave celerity. This criterion was first pointed out by Lighthill and Whitham (1955, p. 294).

The applicability of the kinematic-wave model for the overland-flow problem of a rising hydrograph due to lateral inflow over one plane or channel segment was described by Woolhiser and Liggett (1967) using the kinematic-flow number, $K$; $K$ was defined as:

$$
\frac{\mathrm{S}_{o} L_{o}}{\mathrm{~F}_{o}^{2} y_{o}}=K>10 \text {, }
$$

where $y_{0}=$ normal depth for the maximum steady-state discharge at the end of the flow plane or channel with a maximum rate of lateral inflow,

$\mathrm{F}_{o}=$ Froude number for normal flow $=v_{o} / \sqrt{g y_{o}}$,

$L_{o}=$ length of overland-flow plane or channel segment,

and

$v_{0}=$ steady-state normal flow velocity.

When $K$ is large, the kinematic-wave theory is accurate. When $K=10$, there is approximately 10 percent error in the rising hydrograph from an overland-flow plane. It is noted that $K$ is often much larger than 10 and, therefore, the kinematic-wave model is adequate for most overland-flow problems.

The criterion of Woolhiser and Liggett should only be applied to the rising hydrograph problem where no front face is formed. Because of the continuous lateral inflow in the problem discussed by the authors, the discharge is always greatest at the downstream end of the overland-flow plane or channel segment; therefore, no front face is produced. For applications where a front face is formed due to a change in slope, roughness, or lateral inflow along the plane or channel, the criterion is not applicable because the criterion indicates that a longer channel length is a case where the kinematicwave approximation gives better results. However, for problems that produce a hydrograph with a front face, the further downstream a wave propagates the more the wave has steepended and the less accurate it becomes (fig. 4).

Ponce, Li, and Simons (1978) applied the theory of linear stability to examine the characteristics of dynamic-wave approximations. Following this work, they developed criteria for the application of kinematic-, diffusion-, or dynamic-wave models. For kinematicwave models, they stated that for at least 95-percent accuracy in wave amplitude after one propagation period, the following inequality should hold:

$$
T S_{o}\left(\frac{v}{y}\right) \geq 171
$$

where $T$ is the wave period or length of time required for one wave to pass, and $v$ and $y$ are the mean stream velocity and depth corresponding to an average discharge computed by assuming uniform flow. The amplitude is measured from a mean discharge and after one wave period. For the diffusion-wave model to be an adequate approximation of dynamic waves, the following criterion must be met: 


$$
\mathrm{TS}\left(\frac{g}{y}\right)^{1 / 2} \geq 30 \text {. }
$$

If equation 82 is less than 30 , then the full dynamicwave model must be applied to accurately account for the rate of travel and amount of attenuation.

Henderson (1963) presented the following qualitative criterion based on the bed slope. Flood waves on intermediate slopes are best described by the complete equation of motion (eq. 37 or 78), flood waves on gentle slopes are adequately described by the diffusion-wave model (excluding terms I and II in eq. 78), and flood waves on steep slopes are adequately described by the kinematic-wave model (excluding terms I, II, and III in eq. 78).

The criteria described here for determining the applicability of the different models apply to different situations. Miller and Cunge (1975) showed that the kinematic-wave approximation should not be used for highly supercritical flows. Most overland-flow and channel problems involve subcritical flows and, therefore, the criterion is not generally useful. Woolhiser and Liggett (1967) showed when the kinematic-wave approximation is accurate for simple one-plane or channel applications. This is not a typical application so this criterion is not generally useful. The criteria presented by Ponce, Li, and Simons (1978) are useful for diffusion waves, but, as discussed earlier, because the errors are cumulative, the criterion for kinematic waves is not generally useful. The criteria presented by Henderson (1963) are not quantitative and, therefore, provide only general guidelines.

\section{FURTHER LIMITATIONS OF APPLICABILITY}

Because kinematic waves do not attenuate but always steepen or distort and because flood waves generally do attenuate and often do not steepen significantly, it was suspected that the errors incurred in the kinematic-wave approximation may continuously accumulate. To illustrate the cumulative effect of the errors in the kinematic-wave model, a comparison of solutions using both a kinematic-wave model and a dynamic-wave model was developed.

The comparison was developed for a 100-feet wide rectangular channel with a bed slope, $S_{0}$, equal to 0.01 and a Chézy friction coefficient, $C$, equal to 25 (which is approximately equivalent to a Manning roughness coefficient ranging from 0.060 to 0.068 depending on the depth of flow. A sinusoidal hydrograph was used as input where the wave period, $T$, was equal to 10,000 seconds. The input hydrograph varied from approximately 250 to 700 cubic feet per second. Thus, criteria for the kinematic-wave approximation were checked by the following method. Using equation 81 at a reference flow of $\mathbf{4 0 0}$ cubic feet per second, which results in a depth of 1.38 feet and a mean velocity of 2.90 feet per second.

$$
T S_{0}\left(\frac{v}{y}\right)=210 \geq 171
$$

and, using equation 80 with $L_{o}$ equal to the length of channel traversed in one wave period (wave celerity times wave period),

$$
\frac{S_{0} L_{o}}{F_{o}^{2} y_{o}}=1,628>10
$$

Both of the above criteria are met. The kinematic-wave model should be adequate to predict the propagation of the given wave.

The kinematic-wave model used in the comparison was programmed based on a method of characteristics solution presented by Borah and others (1980), as described in the previous section. However, the shockfitting techniques used by the above authors were not included. Instead, the solution, as described by the kinematic-wave equations alone, was allowed to progress without adjustment for shock formation. If the author's modified technique had been used, a much more accurate result would have been obtained.

The dynamic-wave model programmed for this application is a linear implicit finite-difference model. It is a simple formulation for application to prismatic channels only. It provides a convenient means to test results using different time and distance steps. To minimize the effects of numerical dispersion, the model was run using a Courant number nearly equal to 1 and numerous tests were run using smaller and smaller time and distance steps. Because the model solves a linearized form of the dynamic-wave equations, relatively small time and distance steps were required to minimize the numerical dispersion. The model was further tested by comparing results with a linear implicit finite-difference model developed by Keefer (1976) and documented by Land (1978). The results from these two dynamic-wave models were the same.

Because the downstream boundary condition was not known, an 80,000 -foot channel was modeled for a distance of 200,000 feet. It was assumed that the solution at 80,000 feet would not be affected by the downstream boundary. This assumption was tested using a much shorter reach and only insignificant differences were noted.

The results of the application of kinematic and dynamic models are shown in figure 8 . The input 


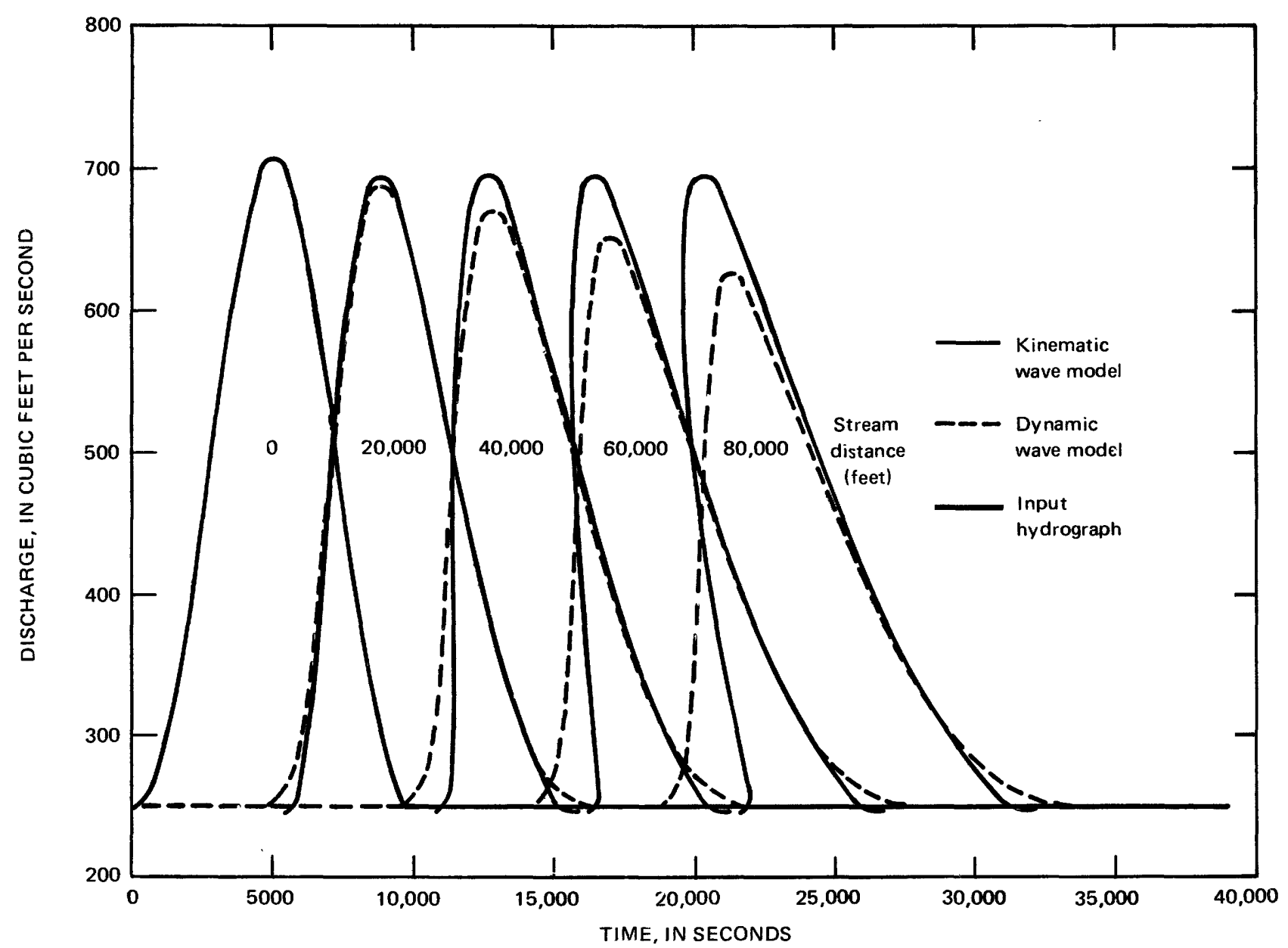

FIGURE 8.-Comparison of kinematic- and dynamic-wave model results showing the upstream hydrograph and predicted hydrographs at $20,000,40,000,60,000$, and 80,000 feet.

hydrograph is shown along with the predicted hydrographs at downstream distances of $20,000,40,000$, 60,000 , and 80,000 feet. The length of the input hydrograph is 37,500 feet. The model was run using a time step equal to 50 seconds and a distance steep equal to 200 feet, giving a Courant number of approximately 1.1. (To compute the Courant number, the wave celerity was determined from the time of travel of the peak discharge.) At a distance of 20,000 feet, the kinematicwave solution shows significant steepening (the change in the discharge is more rapid on the front face of the hydrograph). At a distance of 40,000 feet, kinematic shock has already occurred (more than one discharge is predicted at the same point and time). As the wave moves farther downstream, the wave peak completely overtakes the front edge of the wave. The kinematic-wave model also failed to predict a significant amount of attenuation and dispersion. The criterion for accuracy after one wave period given by Ponce and others (1978) is shown to be approximately correct; however, the errors continue to accumulate as the hydrograph is routed beyond one wave period until the solution becomes unreasonable. The criterion states that the wave amplitude, after one wave period, should be within 95 percent of the correct wave amplitude as compared to some average flow rate and depth. Although the wave meets of all criteria for application of the kinematic-wave model, it can be seen that the error resulting from the kinematic-wave approximations in both the steepening and the attenuation is cumulative as the wave moves downstream.

$\mathrm{Li}$ and others (1976) examined the conditions under which kinematic shock occurs. They showed that along the rising limb of a wave with small lateral inflows, shock will always occur. In a kinematic-wave model, the deeper parts of the wave have a faster wave celerity than the shallow parts. Thus, on the front face or rising limb of the wave, the peak is moving faster than the lower face and the wave continuously steepens until shock occurs. In a dynamic-wave model, attenuation 
and dispersion, although small at each computation step, will generally prevent the steepening of the wave.

Henderson $(1966$, p. 370) stated that as the wave front becomes steeper, the secondary slope terms come into play and introduce dispersion and attenuation, thus delaying or preventing altogether the formation of a surge. However, based on the above comparison of kinematic- and dynamic-wave models for channel-routing problems, the secondary slope terms appear to be significant continuously, and application of a kinematic-wave model to channel problems always gives a steeper wave with less attenuation and dispersion than actually occurs. This result would not be important, however, in applications where there is no formation of a wave front in the channel. An example would be a channel of one slope where the flow originates from evenly distributed lateral inflow.

If a large amount of lateral inflow or channel storage were included in the problem, the error involved in applying the kinematic-wave model would not be as large. Thus, in overland-flow routing where the routing distances are short, the slopes steep, and the lateral inflow large, the kinematic-wave model probably will produce adequate results. However, for most channel-routing applications where the distances are significant and the lateral inflow is not a large percentage of the total flow, the errors accumulate and the kinematic-wave approximation is not adequate. This is true even when the wave meets the criteria which have been set for application of the kinematic-wave approximation. Many channel-routing models are called kinematic-wave models even though they are modified models that include some adjustment to the analytic solution of the kinematic-wave equations, such as numerical dispersion, shock fitting, or adjustments to the bed slope. Many modified models may produce much more adequate results.

\section{SPECIAL TOPICS AND APPLICATIONS}

Three approaches to water routing in which the limitations of kinematic-wave approximations are somewhat relaxed will be presented in this section. These modified kinematic-wave models include the Storm Water Management Model solution, the MuskingumCunge solution, and an implicit four-point finitedifference method.

In the development of the Storm Water Management Model (U.S. Environmental Protection Agency, 1971), it was decided to adjust the friction slope to more adequately describe flow in storm pipes at slopes less than 0.001 . This was done by including terms II and III in addition to term IV in equation 78 to adjust the value of $S_{f}$ for application of the kinematic-wave model. Equation 40 is written, using the Manning equation, as

$$
Q=\frac{1.49}{n} A R^{2 / 3} S_{f}^{1 / 2}
$$

where, from equation 78 ,

$$
S_{f}=S_{0}-\frac{\partial y}{\partial x}-\frac{v}{g} \frac{\partial v}{\partial x}
$$

The continuity equation was solved in a normalized form of equation 6 . The equation was normalized by dividing by $Q$ at pipe-full conditions based on an adjusted friction slope. The friction slope is computed based on the actual flow conditions (not full). Weighting factors $W_{x}$ and $W_{t}$ were used to weight the average of the finite differences at the $i$ and $i+1$ distance steps and the $j$ and $j+1$ time steps. The resulting equation is

$$
\Psi_{i+1, j+1}+C_{1} \alpha_{i+1, j+1}+C_{2}=0,
$$

where

$$
\begin{aligned}
C_{1}= & \frac{\Delta x W_{t} A_{f}}{\Delta t W_{x}\left(Q_{f}\right)_{i+1, j+1}}, \\
C_{2}= & \frac{\Delta x A_{f}}{\Delta t W_{x}\left(Q_{f}\right)_{i+1, j+1}}\left[\left(1-W_{t}\right)\left(\alpha_{i, j+1}-\alpha_{i, j}\right)\right. \\
& \left.-W_{t} \alpha_{i+1, j}\right]+\frac{1-W_{x}}{W_{x}}\left(\psi_{i+1, j}-\psi_{i, j}\right)-\psi_{i, j+1}, \\
\alpha= & \frac{A_{i+1, j+1}}{A_{f}}, \\
\Psi= & \frac{Q_{i+1, j+1}}{\left(Q_{f}\right)_{i+1, j+1}},
\end{aligned}
$$

$A_{f}=$ area of flow at pipe-full conditions,

and

$$
\begin{aligned}
& Q_{f}= \text { discharge at pipe-full conditions, computed } \\
& \text { from equations } 85 \text { and } 86 .
\end{aligned}
$$

Combining equation 85 and 86 results in a nonlinear equation that must be solved using an iterative method (the Newton-Raphson method is suggested in the model documentation). The finite-difference form of equations 85 and 86 is 


$$
\begin{aligned}
\left(Q_{f}\right)_{i+1, j+1}= & \frac{1.49}{n} A_{f} R_{f}^{2 / 3}\left(S_{o}+\frac{y_{i, j-1}-y_{i+1, j-1}}{\Delta x}\right. \\
& \left.+\frac{v_{i, j-1}^{2}-v_{i+1, j-1}^{2}}{2 g \Delta x}\right)^{1 / 2}
\end{aligned}
$$

In the computation scheme, equation 88 is used to compute $Q_{f}$ based on flow conditions at the previous time step. Then equation 87 is solved iteratively. When this scheme was tested, it was found that an iterative procedure to correct values of velocity and depth was needed to prevent oscillations in the solution. Finally, an average value of $\boldsymbol{Q}$ was used based on the last two iterations, in a total of four, applied to each point. In the above formulation, both $W_{x}$ and $W_{t}$ were set equal to 0.55 because it was found that this value provided the best hydrograph peak attenuation and insured stability of the solution. Improved results for pipes with small slopes were obtained with this scheme. Li and others (1976) have presented a similar scheme in which all terms in equation 78 are used to adjust the friction slope. The two schemes described in the Storm Water Management Model and by $\mathrm{Li}$ and others are not simply dynamic- or steady dynamic-wave models as the number of terms included in equation 78 would suggest. Unlike the conventional solution of routing equations where the continuity equation and equation of motion are solved simultaneously, these methods use the additional terms simply to adjust the friction slope used in the kinematic-wave approximation. Some problems have been noted in the application of methods in which $S_{f}$ is adjusted. On the backface of the hydrograph, the additional terms can reduce $S_{f}$ to such an extent that an unreasonable flow is predicted. Therefore, when $S_{f}$ is predicted to be less than $S_{b}$, the solution generally is returned to the kinematic-wave solution for these parts of the hydrograph.

A modified Muskingum method which attempts to quantify the numerical dispersion which results from the fact that the Muskingum method is a finitedifference approximation to the kinematic-wave equations was presented by Cunge (1969). Cunge showed that the determination of the term $D=\frac{q}{S_{o} C \Delta x}$ in the method sets a weighting factor in the finite-difference approximation so that the resulting numerical dispersion approximates the dispersion predicted by the diffusion-wave model. The parameter, $D$, determines the weighting given to the two differences in time, one at the upstream point and one at a distance $\Delta x$ downstream, to approximate the value of $\frac{\partial Q}{\partial t}$ in equation 41. The larger the value of $D$, the greater the amount of dispersion in the solution. The Muskingum-Cunge method with variable parameters is presented here as described by Ponce and Yevjevich (1978). The basic equation is

$$
Q_{i+1, j+1}=C_{1} Q_{i, j}+C_{2} Q_{i, j+1}+C_{3} Q_{j+1, j}
$$

where

$$
\begin{gathered}
C_{1}=\frac{1+C-D}{1+C+D}, \\
C_{2}=\frac{-1+C+D}{1+C+D}, \\
C_{3}=\frac{1-C+D}{1+C+D}, \\
C=\frac{c \Delta t}{\Delta x}=\text { Courant number, }
\end{gathered}
$$

and

$$
D=\frac{q}{S_{o} c \Delta x}
$$

where $c$ and $q$ are the wave celerity and unit width discharge. These values need to be determined for each computation step. Usually, $\Delta t$ is fixed and $\Delta x$ and $S_{o}$ are specified for each channel reach. The values of $c$ and $q$ are determined as follows:

$$
c=\frac{\mathrm{d} Q}{\mathrm{~d} A}
$$

and

$$
q=\frac{Q}{b} .
$$

According to the authors, $\boldsymbol{Q}_{i+1, j+1}$ is computed by iteration. After an initial gues for $\boldsymbol{Q}_{i+1, j+1}$ has been determined by a three-point average of values at grid points $(i, j),(i+1, j)$, and $(i, j+1)$, values for $c$ and $q$ are determined using a four-point average of values of $\boldsymbol{Q}$ and $\boldsymbol{A}$ at grid points $(i, j),(i+1, j),(i, j+1)$, and $(i+1, j+1)$. This iteration is continued until values of $c$ and $q$ have been converged upon. The authors state that this method was shown to be sufficiently accurate for the simulation of flood flows.

A modified kinematic-wave model using an implicit four-point finite-difference method is presented by Alley and Smith (1982, p. 12-15) in a documentation of the recently modified, distributed routing rainfallrunoff model. The scheme is similar to the Muskingum- 
Cunge method in that it attempts to approximate a diffusion-wave model by adjusting the numerical dispersion resulting from the solution technique. The method requires an iterative procedure to solve for the unknown flow area.

In the implicit method, equation 6 is represented by a finite-difference equation using quantities at all four corners of the computational box and a weighting factor for the space derivative. Applying the finitedifference method requires that each model segment be subdivided into distance intervals. A distance interval, $\Delta x$, and a time interval, $\Delta t$, form the computational box for the finite-difference method. The value of $\Delta x$ varies for segment to segment, but the value of $\Delta t$ is constant for all segments.

Four points of a computational box (part of the finitedifference grid in the $x-t$ plane) are represented in figure 9. The purpose of this finite-difference method is to solve $A$ and $Q$ at point $d$, given values of $A$ and $Q$ at points $a, b$, and $c$. This notation for location of points in the finite-difference grid in the $x-t$ plane is inconsis- tent with the notation used in the remainder of this report. It is used here to be consistent with previous documentations of this method such as Dawdy and others (1978). The relationship between the two notations is as follows: $a$ denotes the location $i, j ; b$ denotes the location $i+1, j$; $c$ denotes the location $i, j+1$; and $d$ denotes the location $i+1, j+1$. The equation can be written as

$$
\begin{gathered}
\frac{W_{x}\left(Q_{d}-Q_{c}\right)+\left(1-W_{x}\right)\left(Q_{b}-Q_{a}\right)}{\Delta x} \\
+\frac{\left(A_{d}-A_{b}\right)+\left(A_{c}-A_{a}\right)}{2 \Delta t}=q,
\end{gathered}
$$

where $W_{x}$ is the spatial derivative weighting factor that is assigned a value between 0.5 and 1.0 by the user. The amount of numerical dispersion to be included in the solution is controlled by $W_{x}$. A value of $W_{x}$ near 0.5 will include very little numerical dispersion, resulting in a solution similar to that of the kinematic-wave

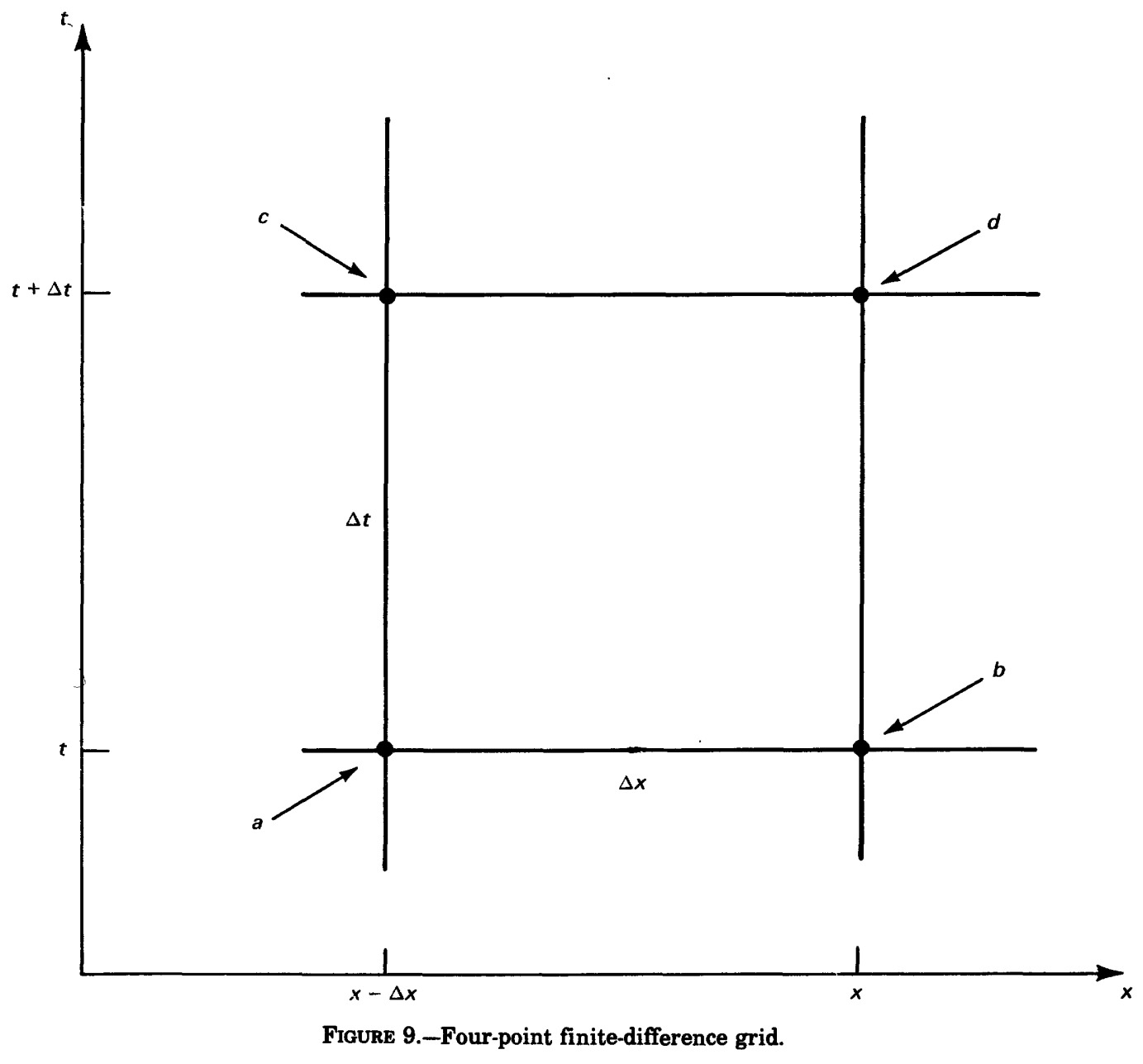


equations. A value of $W_{x}$ near 1.0 will result in a significant amount of dispersion. In this method, the user can control the amount of dispersion in the solution. Whereas no technique for predicting the correct value for $W_{x}$ is given, the value of $W_{x}$ can be determined by calibration or it can be determined based on the suggestions provided in the documentation of the modified distributed routing rainfall-runoff model (Alley and Smith, 1982, p. 33).

Equation 97 has two unknowns, $Q_{d}$ and $A_{d}$, which are related by equation 40 . By substituting $Q_{d}=\alpha\left(A_{d}\right)^{m}$ into equation 96, the resulting equation is nonlinear with one unknown, $A_{d}$, and can be rearranged into the following form:

$$
c_{0}\left(A_{d}\right)^{m}+c_{1} A_{d}+c_{2}=0
$$

where

$$
\begin{gathered}
c_{0}=\alpha, \\
c_{1}=\frac{0.5}{W_{x}} \frac{\Delta x}{\Delta t},
\end{gathered}
$$

and

$$
\begin{gathered}
c_{2}=c_{1}\left[\left(A_{c}-A_{a}\right)-A_{b}\right] \\
+\frac{1-W_{x}}{W_{x}}\left(Q_{b}-Q_{a}\right)-Q_{c}-\frac{q \Delta x}{W_{x}} .
\end{gathered}
$$

The above nonlinear equation for $A_{d}$ is solved with an iterative procedure using Newton's second order method for finding the roots of an equation. The procedure converges rapidly to a correct solution if a good first estimate is made for the unknown area. To speed convergence, the first estimate is obtained by using a modification of the explicit method described under "Supplemental Information" (Dawdy and others, 1978).

Because of errors involved in applying the kinematicwave model to channel problems, a modified scheme, such as one of the three described here, should be used instead. These schemes will help stop the accumulation of errors that occur when the kinematic-wave model is applied.

\section{SUMMARY AND CONCLUSIONS}

Kinematic-wave theory is applicable to overland flow where lateral inflow is continuously added. Where relatively large lateral inflows are involved such as overland-flow problems, and where relatively large overbank storage is considered, the errors resulting from application of the kinematic-wave model may be masked by the overall computational strategy. This is especially true when the routing distances involved are short. However, significant problems occur where the theory is applied to channel routing. In particular, the cumulative error caused by neglecting the secondary slope terms in the equation of motion has been shown.

$\mathrm{Li}$ and others (1976) concluded that the applicability of the kinematic-wave model to river flood routing is very limited because of shock formation. However, it appears that the model is limited in application to channel problems even before kinematic shock occurs. The kinematic-wave model always predicts a steeper wave with less dispersion and attenuation than actually occurs. This only applies, however, to waves that have front faces. The approximations made in the development of the kinematic-wave equations are not generally justified for most channel-routing applications. However, many modified kinematic-wave models can provide adequate results.

\section{REFERENCES}

Abbott, M. B., 1975, Method of characteristics, in Mahmood, K., and Yevjevich, V., eds., Unsteady flow in open channels: Water Resources Publications, p. 63-88.

Alley, W. M., and Smith, P. E., 1982, Distributed routing rainfallrunoff model-Version II: U.S. Geological Survey Open-File Report 82-344, 201 p.

Ames, W. F., 1977, Numerical methods for partial-differential equations: New York, Academic Press, 365 p.

Bakhmeteff, B. A., 1932, Hydraulics of open channels: New York, McGraw-Hill, 329 p.

Borah, D. K., Prasad, S. N., and Alonso, C. V., 1980, Kinematic wave routing incorporating shock fitting: Water Resources Research, v. 16 , no. 3, p. 529-541.

Brakensiek, D. L., 1967, Kinematic flood routing: Transactions of the American Society of Agricultural Engineers, v. 10, no. 3, p. 340-343.

Chow, V. T., 1959, Open-channel hydraulics: New York, McGrawHill, 680 p.

Crandall, S. H., 1956, Engineering analysis: New York, McGrawHill, 417 p.

Cunge, J. A., 1969, On the subject of a flood propagation computation method (Muskingum method): Journal Hydraulic Research, v. 7, no. 2, p. 205-230.

Cunge, J. A. and Woolhiser, D. A., 1975, Irrigation systems, in Mahmood, K. and Yevjevich, V. eds., Unsteady Flow in open channels: Water Resources Publications, p. 509-537.

Dawdy, R. D., Schaake, J. C., and Alley, W. M., 1978, User's guide for distributed routing rainfall-runoff model: U.S. Geological Survey Water-Resources Investigations 78-79, $146 \mathrm{p}$.

Eagleson, P. S., 1970, Dynamic hydrology: New York, McGraw-Hill, $462 \mathrm{p}$.

Gilcrest, B. R., 1950, Flood routing, in Rouse, H., ed., 1949, Engineering Hydraulics: Proceedings of the Fourth Hydraulics Conference, Iowa Institute of Hydraulic Research, chapter 10. 
Gunaratnam, D. J., and Perkins, F. E., 1970, Numerical solution of unsteady flows in open channels: Massachusetts Institute of Technology, Department of Civil Engineering, Hydrodynamics Laboratory Report No. 127, 216 p.

Henderson, F. M., 1963, Flood waves in prismatic channels: Journal of the Hydraulics Division of the American Society of Civil Engineers, v. 89, HY 4, p. 39-67.

1966, Open-channel flow: New York, McMillan, 522 p.

Henderson, F. M., and Wooding, R. A., 1964, Overland flow and ground-water flow from a steady rainfall of finite duration: Journal of Geophysical Research, v. 69, no. 8, p. 1531-1540.

Keefer, T. N., 1976, Comparison of linear systems and finite difference flow routing techniques: Water Resources Research, v. 12, no. 5, p. 997-1006.

Kibler, D. F., and Woolhiser, D. A., 1970, The kinematic cascade as a hydrologic model: Colorado State University, Hydrology Paper No. $39,28 \mathrm{p}$.

Land, L. F., 1978, Unsteady streamflow simulation using a linear implicit finite-difference model: U.S. Geological Survey WaterResource Investigations 78-59, $59 \mathrm{p}$.

Li, R. M., Simons, D. B., and Stevens, M. A., 1975, Nonlinear kinematic wave approximation for water routing: Water Resources Research, v. 11, no. 2, p. 245-252.

Li, R. M., Simons, D. B., Shiao, L. S., and Chen, Y. H., 1976, Kinematic wave approximation for flood routing: Rivers 76; Third Annual Symposium on Inland Waterways for Navigation, Flood Control, and Water Diversion; Colorado State University, v. 1, p. 377-398.

Liggett, J. A., 1975, Basic equations of unsteady flow, in Mahmood, $K$. , and Yevjevich, V., eds., Unsteady flow in open channels: Water Resources Publications, p. 29-62.

Liggett, J. A., and Cunge, J. A., 1975, Numerical methods of solution of the unsteady flow equations, in Mahmood, $\mathbf{K}$., and Yevjevich, V., eds., Unsteady flow in open channels: Water Resources Publications, p. 89-180.

Lighthill, M. J., and Whitham, G. B., 1955, On kinematic waves, I. Flood movement in long rivers: Proceedings of the Royal Society, Series A, v. 229, p. 281-316.

Miller, W. A., and Cunge, J. A., 1975, Simplified equations of unsteady flow, in Mahmood, K., and Yevjevich, V., eds., Unsteady flow in open channels: Water Resources Publications, p. 183-249.

O'Brien, G. G., Hyman, M. A., and Kaplan, S., 1950, A study of the numerical solution of partial-differential equations: Journal of Mathematics and Physics, v. 29, no. 4, p. 223-251.

Ponce, V. M., Li, R. M., and Simons, D. B., 1978, Applicability of kinematic and diffusion models: Journal of the Hydraulic Division of the American Society of Civil Engineers, v. 104, HY 3, p. 353-360.

Ponce, V. M., and Simons, D. B., 1977, Shallow wave propagation in open-channel flow: Journal of the Hydraulics Division of the American Society of Civil Engineers, v. 103, HY 12, p. 1461-1476.

Ponce, V. M., and Yevjevich, V., 1978, Muskingum-Cunge method with variable parameters: Technical Note, Journal of the Hydraulics Division of the American Society of Civil Engineers, v. 104, HY 12, p. $1663-1667$.

Roache, P. J., 1976, Computational fluid dynamics: Albuquerque, N. Mex., Hermosa Publishers, 446 p.

Schaake, J. C., 1965, Synthesis of inlet hydrograph: Johns Hopkins University, $\mathbf{P h}$. D. Dissertation.

1970, Modeling urban runoff as a deterministic process, in Treatise on Urban Water Systems: Proceedings, Institute on Urban Water Systems, Fort Collins, Colo., chap. VI-A p. 343-383.

Theurer, F. D., 1975, A solution for unsteady open-channel flow: Colorado State University, Ph. D. Dissertation, 127 p.

U.S. Environmental Protection Agency, 1971, Storm water manage- ment model: Water Pollution Control Research Series 11024 DOC 07/71, v. 1-Final Report, p. 113-135.

Viessman, W., Knapp, J. W., Lewis, G. L., and Harbaugh, T. E., 1977, Introduction to hydrology: New York, Harper and Row, 704 p.

Weinmann, P. E., and Laurenson, E. M., 1979, Approximate flood routing methods: a review: Journal of the Hydraulics Division of the American Society of Civil Engineers, v. 105, HY 12, p. 15211536.

Woolhiser, D. A., and Liggett, J. A., 1967, Unsteady, one-dimensional flow over a plane-the rising hydrograph: Water Resources Research, v. 3, no. 3, p. 753-771.

Yen, B. C., 1973, Open-channel flow equations revisited: Journal of the Engineering Mechanics Division of the American Society of Civil Engineers, v. 99, EM5, p. 979-1009.

1979, Unsteady flow mathematical modeling techniques, in Shen, H. W., ed., Modeling of rivers: New York, John Wiley, p. 13-1 to 13-33.

Yevjevich, Vujica, 1975, Introduction, in Mahmood, K., and Yevjevich, V., eds., Unsteady flow in open channels: Water Resources Pub. lications, p. 1-27.

\section{SUPPLEMENTAL INFORMATION}

\section{EQUATION DEVELOPMENT CONCEPTS}

The development of the continuity equation and the equation of motion requires the use of some basic concepts. These concepts include the relationship between momentum and energy, the different coordinate systems that can be used, and some friction-slope approximations.

\section{MOMENTUM VERSUS ENERGY}

The development of the equation of motion can be based on Newton's second law (eq. 1) directly, or it can be based on the conservation of energy or of momentum. The relationship between equation 1 , the concept of conservation of energy, and the concept of conservation of momentum is as follows. If both sides of equation 1 are multiplied by a length parallel to the direction of the force and the acceleration, the energy equation is obtained. It states that the work done as a body moves a given length is equal to the kinetic energy acquired by that body. If both sides of equation 1 are multiplied by an elapsed time period, the momentum equation is obtained. It states that the impulse, or force multiplied by time, applied to a body is equal to the momentum, or mass multiplied by velocity (mass times acceleration multiplied by time equals mass times velocity), acquired by it.

The equation of motion is often called the momentum equation. However, because it can be derived based on conservation of momentum or energy, it is not clearly a momentum equation. Chow (1959, p. 50-52) discussed the relationship between the energy and momentum equations for steady, gradually varied flow. He showed 
that the two equations can be practically the same when applied to certain flow problems. However, the equations use different velocity distribution coefficients, although they are nearly equal, and the equations involve different meanings of the frictional losses. In the energy equation, the frictional losses are determined by the internal energy dissipated in the whole mass of water in the reach. In the momentum equation, the frictional losses are determined by the losses due to external forces exerted on the water by the walls of the channel. Yen (1973) showed the relationship between the one-dimensional equation of motion described in this report (Saint Venant equation) and the general momentum and energy equations for open-channel flow. Yen derived the general equations from the Navier-Stokes equations. He stated that the Saint Venant equation is a simplification and approximation of the general equations. However, he also stated that there is no simple way to reduce the general energy equation into the Saint Venant form without making serious and questionable assumptions. Therefore, the equation of motion is probably more clearly a momentum equation than an energy equation. To avoid confusion on this point, the term "equation of motion" is used in this report to describe the Saint Venant equation.

\section{COORDINATE SYSTEMS}

The continuity equation and the equation of motion can be developed using either "gravity oriented" or "natural" coordinate systems. In the gravity-oriented system the $x$-axis is along the horizontal direction of the channel, and in the natural system the $x$-axis is along the longitudinal direction of the channel (at an angle to the horizontal equal to the bed slope). Both systems are orthogonal and are equally acceptable. Some authors have used nonorthogonal systems, for example with the $x$-axis horizontal and depth normal to the channel bed instead of vertical. However, nonorthogonal coordinate systems can result in flow equations that are rather complicated and less suitable for practical purposes (Yen, 1979, p. 13-3).

\section{FRICTION-SLOPE APPROXIMATIONS}

Two friction-slope relationships, which are derived from steady uniform and steady nonuniform flow assumptions, are required for the development of the equation of motion. Because these relationships are not developed based on the more general unsteady nonuniform flow assumptions, they are approximations. However, they are used in the unsteady flow equations so that the friciton slope and the longitudinal shear stress can be expressed using the well-known Chézy formula. This approach is used because no similar relationship exists for unsteady flow.

The Chézy formula is

$$
v=C R^{1 / 2} S_{f}^{1 / 2} .
$$

This equation was developed empirically for steady uniform flow. However, a relationship for $S_{f}$ is developed for application to the unsteady nonuniform flow case by simply rearranging equation 101 as follows:

$$
S_{f}=\frac{v^{2}}{C^{2} R} \text {. }
$$

The acceleration term used in the development of the equation of motion is given in equation 16 . However, for the steady flow situation the derivative with respect to time in the acceleration term is zero so that:

$$
a=v \frac{\mathrm{d} v}{\mathrm{~d} x} .
$$

Substituting equations 12,14 , and 103 into equation 1 ,

$$
-\Upsilon A \Delta h-\tau_{o} P \Delta x \cos \theta=\varrho A \Delta x\left(v \frac{\mathrm{d} v}{\mathrm{~d} x}\right) .
$$

Rearranging and assuming that $\theta$ is small so that $\cos \theta \cong 1$,

$$
\tau_{o}=-\varrho \frac{A}{P} v \frac{\mathrm{d} v}{\mathrm{~d} x} \Upsilon \frac{\mathrm{A}}{P} \frac{\Delta h}{\Delta x} .
$$

Substituting $R=\frac{A}{P}$ and taking the limit as $\Delta x \rightarrow 0$ so that $\frac{\Delta h}{\Delta x}=\frac{\mathrm{d} h}{\mathrm{~d} x}$ and noting that $\Upsilon=\mathrm{eg}$,

$$
\tau_{0}=-\Upsilon R\left(\frac{v}{g} \frac{\mathrm{d} v}{\mathrm{~d} x}+\frac{\mathrm{d} h}{\mathrm{~d} x}\right)
$$

Substituting equation 24 into the previous equation,

$$
\tau_{o}=\Upsilon R S_{f} .
$$

Rearranging, a second relationship for $S_{f}$ is obtained.

$$
S_{f}=\frac{\tau_{o}}{\Upsilon R}
$$

Setting equation 108 equal to equation 25 ,

$$
S_{f}=\frac{\tau_{o}}{\Upsilon R}=\frac{v^{2}}{C^{2} R}
$$


and, rearranging, $\tau_{o}$ is expressed using the Chézy formula:

$$
\tau_{0}=\Upsilon R\left(\frac{v^{2}}{C^{2} R}\right)
$$

\section{METHODS FOR ESTIMATING' $\alpha$ AND $m$}

The parameters $\alpha$ and $m$ are used to define the relationship between flow discharge, $Q$, and flow area, $A$, in the kinematic-wave routing scheme. The following equation is used:

$$
Q=\alpha A^{m}
$$

The $\log$ form of equation 40 is $\log Q=\log \alpha+m \log A$. This is a linear relation in which the intercept is $\log \alpha=\log Q$ when $A=1$, and $m$ is the slope of the relation. If cross-section properties and stage versus discharge data are known for a given channel, $\alpha$ and $m$ can be determined from a $\log -\log$ plot of $Q$ and $A$ as shown in figure 10. For the example data shown in figure $10, \alpha=1.20$ and

$$
m=\frac{\log 9.5-\log 2.5}{\log 5.0-\log 1.8}=1.31
$$

If enough channel data are available to define the relationship between wetted perimeter, $P$, and flow area, $A$, then the following procedure can be used to estimate $\alpha$ and $m$. A log-log plot of $P$ and $A$, similar to that shown in figure 10, can be used to define $a$ and $b$ in the following equation:

$$
P=a A^{b} .
$$

Then equations for estimating $\alpha$ and $m$ can be devised, following the steps below:

$$
R=\frac{A}{P}=\frac{A}{a A^{b}},
$$

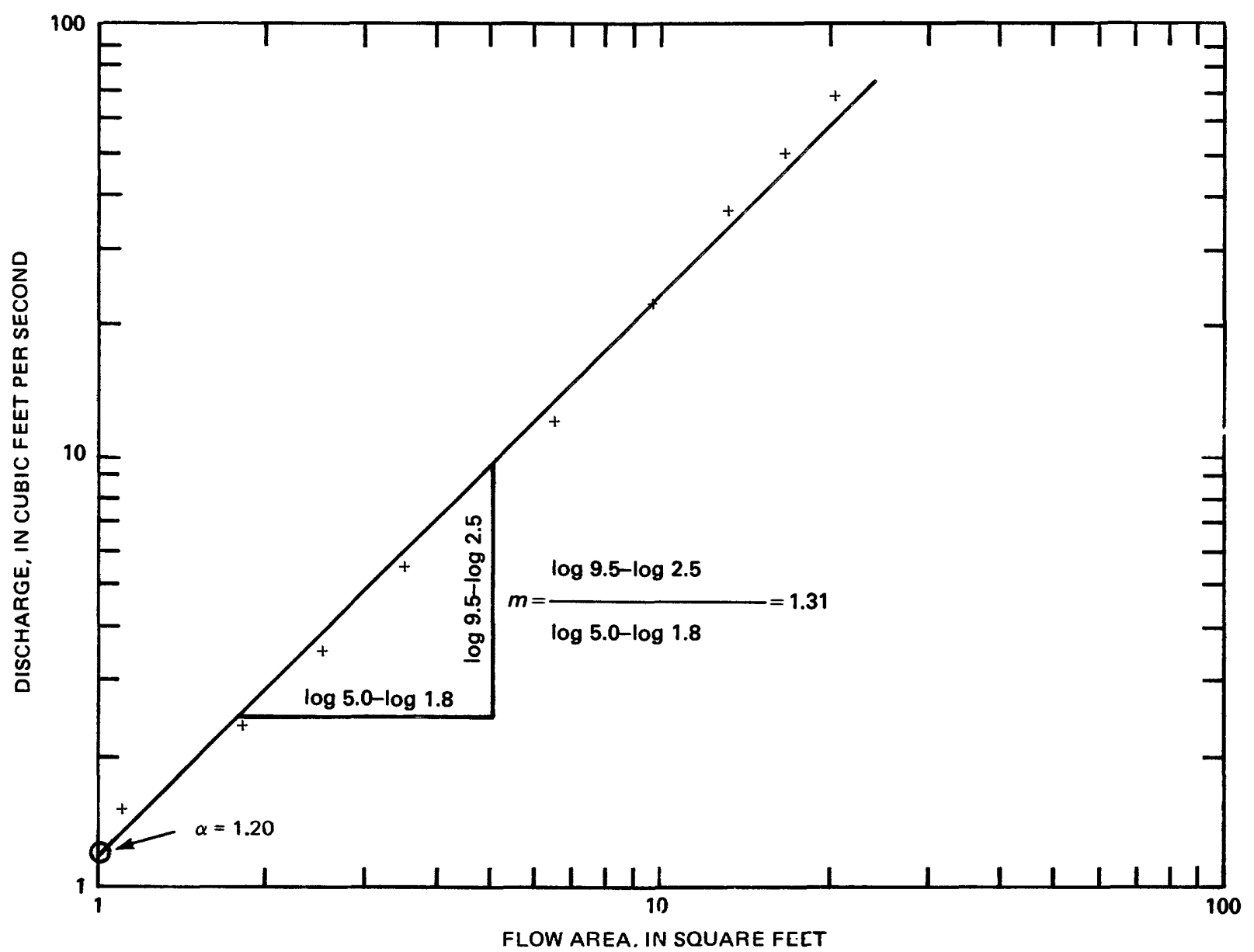

Fiaure 10.-Use of $\log$ - $\log$ plot of discharge, $Q$, and flow area, $A$, to define the routing parameters $\alpha$ and $m$. 
where $R=$ hydraulic radius. Substituting into Manning's formula,

$$
Q=\frac{1.49}{n} A\left(\frac{A}{a A^{b}}\right)^{2 / 3} S^{1 / 2}
$$

and, rearranging,

$$
Q=\frac{1.49 S^{1 / 2}}{n a^{2 / 3}} A^{(5 / 3-2 / 3 b)} .
$$

From equation 114 it can be seen that $\alpha$ and $m$ in equation 40 are

$$
\alpha=\frac{1.49 S^{1 / 2}}{n a^{2 / 3}}
$$

and

$$
m=5 / 3-2 / 3 b \text {. }
$$

The distributed routing rainfall-runoff model (Dawdy and others, 1978, p. 14) provides some approximate relations for determining $\alpha$ and $m$ for channel shapes that can be described as rectangular, trapezoidal, circular, or triangular cross sections. These approximations are given in table 1.

\section{DEVELOPMENT OF DIFFUSION-ANALOGY MODEL}

The diffusion-analogy model is based on the diffusionwave approximation of the dynamic-wave model and the continuity equation. From equation 78 the form of the equation of motion used in the diffusion-wave model is

$$
\frac{\partial y}{\partial x}+S_{f}-S_{b}=0
$$

or

$$
S_{f}=S_{o}-\frac{\partial y}{\partial x}
$$

This is solved simultaneously with the continuity equation as follows (from eq 8):

$$
\frac{\partial Q}{\partial x}+B \frac{\partial y}{\partial t}=0 \text {. }
$$

In this development, the Chézy formula will be used to describe the friction slope. Therefore,

$$
Q=C A\left(R S_{f}\right)^{1 / 2}
$$

and, for wide channels, $A \cong B y$ and $R \cong y$ so that, following equation $120, Q=C B y^{3 / 2} S_{f}^{1 / 2}$ or $v=C\left(y S_{f}\right)^{1 / 2}$. Substituting equation 118 into 120 ,

$$
Q=C A\left[R\left(S_{b}-\frac{\partial y}{\partial x}\right)\right]^{1 / 2}
$$

for wide channels,

$$
Q=C B y^{3 / 2}\left(S_{o}-\frac{\partial y}{\partial x}\right)^{1 / 2} .
$$

Differentiating with respect to $x$, equation 122 becomes

$$
\begin{aligned}
& \frac{\partial Q}{\partial x}=3 / 2 C B y^{1 / 2} \frac{\partial y}{\partial x}\left(S_{o}-\frac{\partial y}{\partial x}\right)^{1 / 2} \\
& -1 / 2 C B y^{3 / 2}\left(S_{b}-\frac{\partial y}{\partial x}\right)^{-1 / 2} \frac{\partial^{2} y}{\partial x^{2}} .
\end{aligned}
$$

Substituting into the continuity equation (eq. 119),

$$
\begin{gathered}
\frac{\partial y}{\partial t}+3 / 2 C y^{1 / 2} \frac{\partial y}{\partial x}\left(S_{0}-\frac{\partial y}{\partial x}\right)^{1 / 2} \\
-1 / 2 C y^{3 / 2}\left(S_{0}-\frac{\partial y}{\partial x}\right)^{-1 / 2} \frac{\partial^{2} y}{\partial x^{2}}=0 .
\end{gathered}
$$

Recalling that $S_{f}=S_{o}-\frac{\partial y}{\partial x}$ and $v=C\left(y S_{f}\right)^{1 / 2}$ and rearranging,

TABLE 1.-Relations for estimating $\alpha$ and $m$ based on physical characteristics of channel segments

\begin{tabular}{lccl}
\hline \multicolumn{1}{c}{ Type of segment } & $\alpha$ & $m$ & Definition of channel parameter $P_{c}$ \\
\hline $\begin{array}{l}\text { Wide rectangular or trapezoidal } \\
\text { cross section }\end{array}$ & $\frac{1.49 S_{o}^{1 / 2}}{n\left(P_{c}\right)^{2 / 3}}$ & 1.67 & $\begin{array}{l}\text { Width of conduit at about mean } \\
\text { depth of flow. }\end{array}$ \\
Circular pipe & $\frac{1.49}{n} \frac{\left(P_{c}\right)^{2 / 3}}{4} S_{o}^{1 / 2}$ & 1.0 & Diameter of pipe. \\
Triangular cross section - & $\frac{1.41 S_{o}^{1 / 2}}{n\left(P_{c}\right)^{1 / 3}}$ & 1.33 & Width at 1-foot depth.
\end{tabular}




$$
\frac{\partial y}{\partial t}+3 / 2 v \frac{\partial y}{\partial x}=\frac{C y^{3 / 2}}{2 S^{1 / 2}} \frac{\partial^{2} y}{\partial x^{2}} \text {. }
$$

Multiplying by $S_{f}^{1 / 2} / S_{f}^{1 / 2}$,

$$
\frac{\partial y}{\partial t}+3 / 2 v \frac{\partial y}{\partial x}=\frac{C y^{3 / 2} S_{f}^{1 / 2}}{2 S_{f}} \frac{\partial^{2} y}{\partial x^{2}}
$$

or, again substituting $v=C\left(y S_{f}\right)^{1 / 2}$,

$$
\frac{\partial y}{\partial t}+3 / 2 v \frac{\partial y}{\partial x}=\frac{v y}{2 S_{f}} \frac{\partial^{2} y}{\partial x^{2}} \text {. }
$$

It has been shown that for kinematic waves, when using the Chézy formula, $c=3 / 2 v$, and choosing the diffusion coefficient to be

$$
\mu=\frac{v y}{2 S_{f}},
$$

equation 127 becomes

$$
\frac{\partial y}{\partial t}+c \frac{\partial y}{\partial x}=\mu \frac{\partial^{2} y}{\partial x^{2}}
$$

This equation is the diffusion equation and in the application to flow routing is called the diffusion analogy. A diffusion equation with discharge instead of depth has as the dependent variable also can be developed based on some additional assumptions.

\section{DEVELOPMENT OF STEP-BACKWATER FROM DYNAMIC-WAVE MODEL}

The development of the step-backwater procedure is given to show that it is one of the many approximations of the dynamic-wave model. The step-backwater procedure is the standard-step method of computing steady, gradually varied flow as described by Chow (1959, p. 265-268). The dynamic-wave model includes the continuity equation and the equation of motion as follows:

$$
\frac{\partial Q}{\partial x}+B \frac{\partial y}{\partial t}=0
$$

and

$$
S_{f}=\frac{v^{2}}{C^{2} R}=S_{b}-\frac{\partial y}{\partial x}-\frac{v}{g} \frac{\partial v}{\partial x}-\frac{1}{g} \frac{\partial v}{\partial t}
$$

Following the assumption of steady flow, all terms describing the variation with respect to time are zero. Thus, equations 119 and 36 can be rearranged and sim- plified to ordinary differential equations describing only variation with respect to $x$ :

$$
\frac{\mathrm{d} Q}{\mathrm{~d} x}=0
$$

and

$$
\frac{v}{g} \frac{\mathrm{d} v}{\mathrm{~d} x}+\frac{\mathrm{d} y}{\mathrm{~d} x}+S_{f}-S_{0}=0
$$

Equation 129 simply states that the discharge does not vary with distance. This is true within each computation of the step-backwater procedure. The remainder of the development is concerned with equation 130 . Recalling that

$$
\frac{\mathrm{d} v^{2}}{\mathrm{~d} x}=v \frac{\mathrm{d} v}{\mathrm{~d} x}+v \frac{\mathrm{d} v}{\mathrm{~d} x}=2 v \frac{\mathrm{d} v}{\mathrm{~d} x},
$$

the first term in equation 130 can be rearranged as follows:

$$
\frac{v}{g} \frac{\mathrm{d} v}{\mathrm{~d} x}=\frac{1}{2 g} \frac{\mathrm{d} v^{2}}{\mathrm{~d} x}=\frac{\mathrm{d}\left(\frac{v^{2}}{2 g}\right)}{\mathrm{d} x} .
$$

Therefore, equation 130 becomes

$$
\frac{\mathrm{d}\left(\frac{v^{2}}{2 g}\right)}{\mathrm{d} x}+\frac{\mathrm{d} y}{\mathrm{~d} x}+S_{f}-S_{b}=0 \text {. }
$$

Approximating the differentials with a simple finite difference in which the subscript 1 refers to a channel cross section at some distance $x$ and subscript 2 refers to a section a distance $\Delta x$ downstream,

$$
\frac{\frac{\left(v_{1}\right)^{2}}{2 g}-\frac{\left(v_{2}\right)^{2}}{2 g}}{\Delta x}+\frac{y_{1}-y_{2}}{\Delta x}+S_{f}-S_{o}=0 .
$$

Multiplying by $\Delta x$ and rearranging,

$$
\frac{\left(v_{1}\right)^{2}}{2 g}-\frac{\left(v_{2}\right)^{2}}{2 g}+y_{1}-y_{2}=\left(S_{o}-S_{f}\right)(\Delta x)=S_{o} \Delta x-S_{f} \Delta x .
$$

The friction slope can be written as

$$
S_{f}=\frac{-h_{f}}{\Delta x},
$$

where $h_{f}=$ friction loss.

The bed slope can be written as

$$
S_{0}=\frac{-\Delta z}{\Delta x}=\frac{-\left(z_{1}-z_{2}\right)}{\Delta x} .
$$


Substituting equations 136 and 137 into equation 135,

$$
\frac{\left(v_{1}\right)^{2}}{2 g}-\frac{\left(v_{2}\right)^{2}}{2 g}+y_{1}-y_{2}=z_{2}-z_{1}+h_{f}
$$

Rearranging,

$$
\frac{\left(v_{1}\right)^{2}}{2 g}+y_{1}+z_{1}=\frac{\left(v_{2}\right)^{2}}{2 g}+y_{2}+z_{2}+h_{f}
$$

Equation 130 is the basis of the step-backvrater procedure. Usually, an estimate of the velocity distribution coefficient at both sections and an estimate of the eddy losses is included in a step-backwater procedure. However, they are missing here because they were ignored in the development of the unsteady flow model and are often ignored in practical applications of unsteady flow models.

\section{KINEMATIC-WAVE MODEL USED IN THE DISTFIBUTED ROUTING RAINFALL-RUNOFF MODEL}

The kinematic-wave equations are solved using a finite-difference approximation. The explicit scheme used is generally stable for any channel segment length, $x$, and any time step, $t$. There are some exceptions to this when there is a large inflow at the initial time.

The continuity equation to be solved using a finitedifference scheme is

$$
\frac{\partial A}{\partial t}+\frac{\partial Q}{\partial x}=q
$$

The relationship between $A$ and $Q$ is approximated by

$$
Q=\alpha A^{m} \text {. }
$$

The model computes $\alpha$ and $m$ for channel shapes that can be approximated by rectangular, trapezioidal, circular, or triangular cross sections. These approximations are given in table 1.

A rectangular grid of points is used to approximate $Q$ and $A$ at the downstream end of the channell using $Q$ and $A$ at the upstream end of the channel to drive the computations. The notation used here is consistent with previous documentations of this method as described under "Special Topics and Applications". Figure 9 shows the four-point finite-difference grid used to solve for $Q$ and $A$ at point $d$-the downstream $\in$ ind of the channel segment at the next time step. Points $a$ and $b$ are the upstream and downstream ends of the channel segment at the present time step and point $c$ is the upstream end of the channel at the next time step. $Q$ and $A$ are known at points $a, b$, and $c$ at every time step.

In an attempt to maintain a generally stable solution, the model contains two different finite-difference equations and selects the appropriate one at each point in the solution. The decision depends on the parameter.

$$
\theta=m \frac{\Delta t}{\Delta x} \frac{Q_{b}}{A_{b}}=\frac{\text { kinematic wave speed }}{\Delta x / \Delta t} .
$$

The parameter, $\theta$, determines whether the characteristic path passing through point $d$ in figure 9 passes above or below point $a$. If $\theta$ is greater than or equal to unity, the water wave has traveled through the entire reach, $\Delta x$, during $\Delta t$ and the finite-difference equations are written across time between points $a$ and $c$. The continuity equation is approximated as

$$
\frac{A_{c}-A_{a}}{\Delta t}+\frac{Q_{d}-Q_{c}}{\Delta x}=q .
$$

Rearranging, the equations are

$$
\boldsymbol{Q}_{d}=Q_{c}+q \Delta x-\frac{\Delta x}{\Delta t}\left(A_{c}-A_{a}\right)
$$

and

$$
A_{d}=\left(Q_{d} / \alpha\right)^{1 / m}
$$

This involves only grid points $a, c$, and $d$. If $\theta$ is less than unity, the water has traveled a distance less than the reach length, $\Delta x$, during $\Delta t$ and the finite-difference equations are written across space between points $a$ and $b$. The continuity equation is approximated as

$$
\frac{A_{d}-A_{b}}{\Delta t}+\frac{Q_{b}-Q_{a}}{\Delta x}=q
$$

Rearranging, the equation are

$$
A_{d}=A_{b}+q \Delta t+\frac{\Delta t}{\Delta x}\left(Q_{a}-Q_{b}\right)
$$

and

$$
Q_{d}=\alpha\left(A_{d}\right)^{m}
$$

